\title{
The dynamical parameters of turbulence theory as they apply to middle atmosphere studies
}

\author{
W. K. Hocking
}

The University of Western Ontario, London, Ont., Canada

(Received August 21, 1998; Revised January 20, 1999; Accepted April 12, 1999)

\begin{abstract}
The study of turbulent heating and diffusion in the middle atmosphere is complicated by some subtle points relating to the application of existing theory. Incorrect interpretation of turbulent spectra can result, leading to errors in estimates of the strengths of turbulence by factors of 5 and more. In this short review, the relevant turbulent spectra and equations are considered, and their applications in middle atmosphere studies are outlined. New developments with regard to some of this theory, and especially new understandings about the dynamical parameters used in some of these applications (often referred to as the "constants" of the equations) are described. Current areas of uncertainty are also considered, both in relation to turbulent energy dissipation as well as diffusion over various scales.
\end{abstract}

\section{Introduction}

In studies of turbulence, the optimum spectra to use for calculations of kinetic energy dissipation rates are often the velocity spectra. These are dealt with in some detail in the literature (e.g. Batchelor, 1953; Tatarskii, 1961, 1971). For freely decaying turbulence we can consider $\varepsilon$, the kinetic energy dissipation rate, as

$$
\varepsilon=-\frac{d}{d t} \frac{1}{2} \overline{\left[u^{\prime 2}+v^{\prime 2}+w^{\prime 2}\right]},
$$

where $\overline{\left[u^{\prime 2}+v^{\prime 2}+w^{\prime 2}\right]}$ is the total mean square velocity fluctuation, and $\overline{\frac{1}{2}\left[u^{\prime 2}+v^{\prime 2}+w^{\prime 2}\right]}$ is therefore the mean kinetic energy per unit mass at any instant in time (Batchelor, 1953, page 86). The overbar refers to a spatial average. (An even more fundamental discussion about the energy dissipation rate can be found in Batchelor, 1967, Subsection 3.4, but that is beyond our requirements for this paper.)

If an experimentalist can obtain velocity fluctuations at scales within the inertial range of turbulence, or even into the viscous range, then calculation of kinetic energy dissipation rates is very straight forward. For example, if an observer is dealing with isotropic, homogeneous turbulence, and if that observer can make measurements at scales within the inertial range and deep into the viscous range, then the kinetic energy dissipation rate $\varepsilon$ can be found directly by integrating across the spectrum as

$$
\varepsilon=2 \int_{0}^{\infty} \nu k^{2} E(k)
$$

where $v$ is the kinematic molecular viscosity coefficient, $k$ is the wave number, and $E(k)$ is the spectral density of velocity fluctuations (sum of all three components) over a shell

Copy right $(\mathrm{C})$ The Society of Geomagnetism and Earth, Planetary and Space Science (SGEPSS); The Seismological Society of Japan; The Volcanological Society of Japan; The Geodetic Society of Japan; The Japanese Society for Planetary Sciences. in wave-number space of radius $k$ (e.g. see Batchelor, 1953; Hocking and Hamza, 1997, and references therein). However, in atmospheric sciences, determination of $E(k)$ down to scales this small is rarely possible. In the middle atmosphere, it simply cannot be achieved with current technology.

If it is possible to determine velocity fluctuations down to scales at least into the inertial range, determination of $\varepsilon$ is still modestly easy, although one often needs to make assumptions about the form of turbulence (isotropic, Kolmogoroff theory etc.). Examples of such applications exist in the literature: for example, Barat (1982) has shown how this may be done using structure functions.

However, Barat's measurements required a high altitude balloon, and special instrumentation. Measurements into the upper middle atmosphere by this method are limited by a ceiling on the balloon altitude. In-situ measurements above say $40 \mathrm{~km}$ altitude are limited to rockets, and because these must travel at high speed, they cannot sample the velocities with sufficient resolution to apply such methods.

Measurements of middle atmosphere turbulence are therefore largely limited to radar techniques, and occasional rocket and balloon studies. Within these categories, only special balloon-borne instrumentation is capable of direct velocity measurements at sufficient spatial and temporal resolution to enable direct calculation of $\varepsilon$, and even then high altitude balloons are only flown rarely. All other methods involve measurements of velocity fluctuations which effectively integrate over moderately large intervals of scale, or involve measurements of parameters other than the velocity fluctuations. In the former case, the integration limits and instrumental weighting are often hard to determine, and in the latter case it is often necessary to make various assumptions, and determine other parameters such as background gradients, before turbulence strengths can be calculated.

This review focuses on a critical examination of the assumptions made in developing the formulae which are used in determination of middle atmosphere turbulence strengths, 
and highlights recent developments in this area.

\section{Currently Used Formulae}

The following equations present various formulae which are currently used for determinations of middle atmosphere turbulence strengths.

$$
\begin{gathered}
\varepsilon=c_{0}\left(\sigma^{2}\right)^{3 / 2} / L_{\mathrm{B}}, \\
L_{\mathrm{B}}=\frac{2 \pi}{c_{3}} \varepsilon^{1 / 2} \omega_{\mathrm{B}}^{-3 / 2}, \\
\varepsilon=c_{1}\left(\sigma^{2}\right) \omega_{\mathrm{B}} .
\end{gathered}
$$

Variations of these formulae have been presented by, for example, Weinstock, (1978a,b, 1981), and Hocking (1983, 1986). The term $\omega_{\mathrm{B}}$ is the Väisälä-Brunt frequency, $\varepsilon$ is the kinetic energy dissipation rate, $\sigma$ refers to a typical rootmean-square velocity (to be specified in more detail later), and $L_{\mathrm{B}}$ is a scale related to the larger eddies.

These equations appear deceptively simple, but they are in fact complicated by several factors. Principal amongst these is the fact that the term $\sigma^{2}$ is sometimes ill-defined. The constants $c_{0}$ and $c_{1}$ are critically dependent on how $\sigma^{2}$ is determined. Formulae of this type are often used in both in-situ and radar studies, but the nature of the determination of $\sigma^{2}$ must be very carefully considered. For example, in radar studies it is usually an integral over the radar volume, and over the duration of the radar record used for the calculation. The details of this integration process need to be carefully considered. As we will see, there are also additional complications, and even the choice of the scale $L_{\mathrm{B}}$ is complicated.

In fact there are some references which use yet another variant on Eq. (3). This equation takes the form

$$
\varepsilon=c_{6}\left(\sigma^{2}\right)^{3 / 2} / L_{\mathrm{r}}
$$

where $L_{\mathrm{r}}$ is a scale associated with the radar beam and pulselength, and not the scale $L_{\mathrm{B}}$ defined above (e.g. Labitt, 1979; Bohne, 1982; Doviak and Zrnic, 1984). We therefore need to ask: which of these two options (i.e. Eqs. (3) or (6)) is preferable?

Thus we recognize that these equations, despite a deceptively simple appearance, are not well understood, and we pose the questions: What do we mean by $\sigma^{2}$ ? Which scale " $L$ " should we use? Answering these questions will be one of our responsibilities in this paper.

There are also other equations which appear in the literature which need to be more properly understood. Some such equations are the expressions

$$
\begin{gathered}
\eta=\left(\frac{\nu^{3}}{\varepsilon}\right)^{1 / 4}, \\
\ell_{0}=c_{4} \eta
\end{gathered}
$$

and

$$
L_{\mathrm{B}}=c_{5} L_{0} \text {. }
$$

Again, these are very simple equations, but with hidden complications. We will define the various terms and consider these expressions shortly.
Another expression used in the literature to determine $\varepsilon$, which utilizes the mean square refractive index fluctuation quantity $C_{n}^{2}$, is

$$
\bar{\varepsilon}=\left(\gamma \overline{C_{n}{ }^{2}} \frac{\omega_{\mathrm{B}}^{2}}{F^{1 / 3}} M^{-2}\right)^{3 / 2} .
$$

$C_{n}^{2}$ is often called the "potential refractive index structure constant", although the use of the word "constant" here can be quite misleading, since the quantity is far from constantit in fact varies markedly as a function of the intensity of the turbulence. Nevertheless, we must persist with this usage, since it is very common. However, the reader should bear in mind that $C_{n}^{2}$ is in fact a measure of the amount of refractive index fluctuation in a given turbulent patch, and is not to be considered in the same category as the other dynamical parameters (also referred to as "constants") which are the topic of this paper. Again, the above expression looks simple enough, but application of this expression is complicated by determination of the term " $F$ " (which represents the fraction of the radar volume which is turbulent), and by a proper determination of the "constant" $\gamma$-which in fact turns out to be Richardson-number dependent. We will not consider the factor " $F$ " any further here; our main interest is in the parameter $\gamma$. Discussions relating to " $F$ " can be found in Van Zandt et al. (1978, 1981) and Hocking and Mu (1997).

Finally, there is another expression which appears to be exquisitly simple, yet hides a multitude of complexity. This is the expression

$$
K=c_{2} \frac{\varepsilon}{\omega_{\mathrm{B}}^{2}}
$$

where $K$ represents a diffusion coefficient. This relation purports to relate the rates of atmospheric diffusion and the value of the kinetic energy dissipation rate. However, it raises many issues. It may be derived from modestly simple arguments; for example, Fukao et al. (1994), Appendix A, gives one example. However, there are yet further questions about this. Is the derivation too simplistic? Is it valid at all? If it is valid, what should be the "constant" $c_{2}$ ? Different authors have proposed different values for $c_{2}$. If indeed it does apply, is it valid over all scales? If the scale-range is limited, what limits exist? McIntyre (1989) has even considered that the value of $c_{2}$ might depend in some way on the mode of turbulence generation, and the degree of super-saturation of the waves which generate the turbulence. How realistic is this proposal? In that case, it would not even make sense to assume that $c_{2}$ is a constant for an individual event, although there might still be some long-term average value of $c_{2}$ which can be applied to the middle atmosphere. We cannot address all these issues, but will try and consider at least some of them.

Thus, while we recognize that these formulae are used in the literature for determination of $\varepsilon$ and $K$, we also recognize that each equation embodies a complication of one sort or another. A major objective of this paper will be to highlight, and where possible clarify, these complications.

We will begin our discussions by pointing the reader to Appendices $\mathrm{A}$ to $\mathrm{D}$, which contain expressions for the currently accepted structure functions and spectra which are generally used in theoretical Kolmogoroff turbulence studies. In general the formulae are presented without proof: they are meant 
to simply be a summary of the main and simplest tools used in turbulence studies. We begin with functions associated with measurements of velocity, and later move to measurements of tracers and scalars.

In our next sections, we then begin to address some of the questions raised above.

\section{Turbulence Scales and Inverse Scales}

We first turn to a discussion of the Eqs. (7) to (9), mainly because the questions posed in relation to these expressions are some of the simplest to answer.

The first equation, (7), is a derivation produced from dimensional analysis. However, once derived it can be usefully employed as a scaling length. Physically, it is a scale within the viscous range of the turbulence, and represents a typical scale at which energy transfer by scale-cascade, and energy dissipation to heat, are comparable. The scale $\ell_{0}$ is a scale which represents the transition between the inertial and viscous ranges of turbulence, and is defined in terms of the intercept between extrapolations of the spectral forms in these two ranges (e.g. see Tatarskii, 1971). It is always bigger than $\eta$, and the constant $c_{4}$ is in fact fairly well known. However, it is important to recognize that even $c_{4}$ depends on whether one is using measurements of velocity fluctuations or some sort of constituent or tracer. Typical values of $c_{4}$ are 7.4 for temperature fluctuations (e.g. Hill and Clifford, 1978), and $(15 C)^{3 / 4}$ (where $C=2.0$ ), or 12.8 , for velocity fluctuations (e.g. Tatarskii, 1961).

Thus these scales are at least fairly well understood, although on occasions some authors have assigned them to have units of metres per radian, which is wrong. They are simply units of length.

Equation (9) does introduce some extra complications, however, which sometimes lead to confusion. The scale $L_{0}$ is a vertical scale at which the RMS fluctuations due to the turbulence are equal to the change in the mean value of the same quantity over the same vertical scale. This is quite different to $L_{\mathrm{B}}$, which is a scale at the "large-scale end" of the inertial range of the spectrum. The latter quantity is usually much larger than the first-often by more than an order of magnitude (e.g. see Hocking, 1985, who gives a ratio in the order of 30).

To complicate things further, an alternative scale to $L_{\mathrm{B}}$ is often used, which equals $\varepsilon^{1 / 2} \omega_{\mathrm{B}}^{-3 / 2}$ and is called the Ozmidov scale. This differs from $L_{\mathrm{B}}$ only by a multiplicative constant of $2 \pi / c_{3}$, so conceptually is very similar to $L_{\mathrm{B}}$. We will generally use $L_{\mathrm{B}}$, since this has become more common in middle atmosphere work, and Barat (1982) has shown that it does indeed seem to relate fairly nicely to the low wavenumber end of the inertial range.

Another common problem which occurs in discussions about the scales of turbulence is the use of inverse scaling factors. Whilst a scale is assigned a "wavelength" $\lambda$, and its corresponding wavenumber is $k=2 \pi / \lambda$, it is not uncommon to use special inverse scales which relate to particular spatial lengths by a simple reciprocal relation. For example, sometimes a scale $k_{\mathrm{B}}^{*}=1 / L_{\mathrm{B}}$ is used for scaling purposes. This seems at odds with the wavenumber $k_{\mathrm{B}}=2 \pi / L_{\mathrm{B}}$, but in fact there is no conflict; we will therefore dispense with this issue here. $L_{\mathrm{B}}$ is a "typical" scale, but does not particularly represent the distance between the maxima of any special sinusoidal fluctuation. Therefore there is no obligation to use $2 \pi$ as the scaling constant, so $k_{\mathrm{B}}^{*}=1 / L_{\mathrm{B}}$ is just as useful for scaling purposes as $2 \pi / L_{\mathrm{B}}$. Problems arise, however, when $k_{\mathrm{B}}^{*}$ is referred to as a "wavenumber"; it is in fact not one, and should be considered (when used) as nothing more than an inverse scaling factor. Confusion arises because scaling parameters like this are sometimes referred to as "wavenumbers", and because they are often denoted by symbols which are traditionally used for harmonic quantities. If, on the other hand, one is talking of true wavenumbers, and their relation to "wavelengths", then one must use $k=2 \pi / \lambda$.

\section{Relation between $\varepsilon$ and $\sigma^{2}$}

In this section, we wish to address the issue of the correct relation between $\sigma^{2}$ and $\varepsilon$, as described in Eqs. (3) and (6). The equations look similar, but in fact are very different conceptually, and we need to understand why.

In studies of turbulence with a radar, one usually measures a complex-amplitude time-series which is a result of radio-wave scatter from a region of space called the "radar volume". This volume is defined by the radar beam and radar pulse-length. Within this volume, scatterers are moving with a variety of velocities, and the observed signal is due to a combination of Doppler shifted echoes produced when the radiowaves scatter from these entities. The received signal can be Fourier transformed to produce a spectrum, which has a half-power half-width of $f_{1 / 2}$, and an associated variance $f_{v}^{2}$. If we multiply $f_{v}^{2}$ by $(\lambda / 2)^{2}$, where $\lambda$ is the radar wavelength, then we produce a variance in terms of velocity units, which we denote as $\sigma^{2}$. This variance is an integrated effect of all the velocity fluctuations within the radar volume, as shown diagrammatically by Hocking (1983).

Detailed derivations of the relation between the turbulence velocity spectrum (which describes the fluctuations inside the radar volume) and the value of $\sigma^{2}$ have been presented by (amongst others) Hocking (1983), Labitt (1979), Bohne (1982) and Hocking (1996a). In the following subsections, we will briefly re-visit some of these derivations.

To begin, we will follow the derivation presented by Hocking (1983), which produces Eq. (3).

\subsection{Buoyancy scale dependence between $\sigma^{2}$ and $\varepsilon$}

Assuming a Kolmogoroff form for the turbulence spectrum, Hocking $(1983,1986)$ has shown that

$$
\sigma^{2} \propto \int_{2 \pi / L_{\mathrm{B}}}^{k=4 \pi / \lambda} \varepsilon^{2 / 3} k^{-5 / 3} d k,
$$

where $\sigma$ is the root mean square velocity deduced from the spectral width of the signal. At this stage we will not concern ourselves with the constants of proportionality; our main interest here is in the general form of the equation. We will shortly produce a more sophisticated form of this equation in which the relevant constants of proportionality will become clearer. This equation expresses the fact that the velocity variance measured by the radar is the integrated effect of different scales within the radar volume.

Upon integration we obtain the following expression:

$$
\sigma^{2} \propto \varepsilon^{2 / 3}\left[L_{\mathrm{B}}^{2 / 3}-\left[\frac{\lambda}{2}\right]^{2 / 3}\right]
$$


Assuming that $L_{\mathrm{B}} \gg \lambda / 2$ we have the following relationship:

$$
\varepsilon=c_{1} \frac{\sigma^{3}}{L_{\mathrm{B}}} .
$$

The value of $c_{1}$ differs somewhat for different assumptions about the constants involved in the Kolmogoroff spectrum, but Hocking (1983) has given a value of $c_{1}$ of 3.5. This value assumes that the fluctuations producing the radar signal are produced in roughly equal proportion by scales in the buoyancy range and the inertial range. We shall re-address this assumption shortly.

If, in addition, we use the relation between the buoyancy scale $L_{\mathrm{B}}$ and the Väisälä-Brunt frequency which was specified earlier as

$$
L_{\mathrm{B}}=\frac{2 \pi}{0.62} \varepsilon^{1 / 2} \omega_{\mathrm{B}}^{-3 / 2}
$$

(Weinstock, 1978b), we may then write

$$
\varepsilon=c_{0} \sigma^{2} \omega_{\mathrm{B}}
$$

where $c_{0}$ is a constant $(\sim 0.45)$.

In contrast to this expression, (which is commonly used in mesospheric and stratospheric radar studies), equations relating radar spectral widths and turbulent energy dissipation rates which have been presented in the meteorological literature have tended to ignore the possibility that the buoyancy scale may play a role in the relation between $\varepsilon$ and $\sigma^{2}$. Rather, they have assumed that either the length of the radar pulse, or the radar beam-width, (whichever is larger) is the most important parameter in determining this $\varepsilon-\sigma^{2}$ relation. We will now look at this particular derivation in more detail.

\subsection{Radar volume dependence between $\sigma^{2}$ and $\varepsilon$}

The following derivation briefly summarizes that presented by Labitt (1979), and also presented by Hocking (1996a). We do not intend to repeat their derivations in detail here, and so we start with the relation

$$
\sigma^{2}=\int_{-\infty}^{\infty} \int_{-\infty}^{\infty} \int_{-\infty}^{\infty} \Phi_{\ell \ell}(\underline{k})\left[1-\mathrm{e}^{-\left[k_{z}^{2} b^{2}+k_{y}^{2} a^{2}+k_{x}^{2} a^{2}\right]}\right] \underline{d k},
$$

which is derived in those references. $\Phi_{i j}$ is described in Eq. (B.4), and in this case we take $i=j$ i.e. both velocity components aligned in the direction parallel to the direction of traverse (in this case the direction of traverse of the radar beam) through the patch of turbulence (see Appendices A to D). The term in square brackets is simply 1 minus the Fourier transform of the radar volume, and therefore takes into account the radar weighting. Equation (17) is in fact similar in some aspects to Eq. (12), but there are also some important differences between the two. The former one essentially assumes that all radial motions are parallel to the bore-sight direction of the radar, while this newer one recognizes that there may be contributions from off-bore-sight components if the beam is broad. Equation (12) also contains no specific radar weighting, but does contain a lower limit on $k$ which is defined by the largest turbulence scales. Equation (17) contains no such turbulence-defined limit, and this will shortly prove to be an important point.

It is also important to point out that neither of these formulae recognize the fact that the velocity spectrum should actually be anisotropic at scales comparable to and larger than $L_{\mathrm{B}}$. However, since for MST work the radars usually point vertically, and it is primarily the vertical velocity spectrum which affects the spectral width, it is only necessary that a reasonable estimate of the vertical velocity spectrum is produced for our work here. In this case, we specify $E(k)$, and the vertical velocity spectrum is derived from that, but we have allowed a reasonable range of possibilities for $E(k)$, and therefore a reasonable range of vertical velocity spectra. The key point is that $E(k)$ is chosen so that the vertical velocity spectra are realistic. Since horizontal fluctuations are of secondary significance for a vertically pointed, narrow beam, the issue of anisotropy is not so crucial here. Hocking (1996a), and Hocking and Hamza (1997) has discussed the issues of anisotropy in a little more detail.

If one then takes the classical inertial range spectrum (e.g. see Tatarskii, 1971), then the spectrum of vertical velocities as a function of wave number $\underline{k}$ is

$$
\Phi_{\ell \ell}(\underline{k})=\frac{E(k)}{4 \pi k^{2}}\left[1-\frac{k_{z}^{2}}{k^{2}}\right],
$$

where $k$ is the magnitude of $k$ and so is a scalar satisfying $k^{2}=k_{x}^{2}+k_{y}^{2}+k_{z}^{2}, E(k)=\alpha \varepsilon^{2 / 3} k^{-5 / 3}$, and $\alpha$ is a numerical constant with value $0.7655 C$, where $C=2.0$ (see Eq. (B.4)).

The following expression for the velocity variance measured by the radar may now be obtained:

$$
\sigma^{2}=\frac{1}{2} \alpha \varepsilon^{2 / 3} \Upsilon
$$

where

$$
\begin{aligned}
\Upsilon= & \int_{\theta=0}^{\pi} \int_{k=0}^{\infty} \sin ^{3} \theta k^{-5 / 3} \\
& \times\left[1-\mathrm{e}^{-k^{2}\left[a^{2} \sin ^{2} \theta+b^{2} \cos ^{2} \theta\right]}\right] d k d \theta
\end{aligned}
$$

Thence

$$
\varepsilon=\frac{2 \sqrt{2}}{[\alpha \Upsilon]^{3 / 2}} \sigma^{3}
$$

Finally, the following expressions for $\Upsilon$ are valid: Firstly if $a \geq b$ :

$$
\Upsilon=2 \Gamma\left(\frac{2}{3}\right) a^{2 / 3} F\left[\frac{-1}{3} ; \frac{1}{2} ; \frac{5}{2} ; 1-\frac{b^{2}}{a^{2}}\right]
$$

whilst if $b \geq a$ :

$$
\Upsilon=2 \Gamma\left(\frac{2}{3}\right) a^{2 / 3} F\left[\frac{-1}{3} ; 2 ; \frac{5}{2} ; 1-\frac{a^{2}}{b^{2}}\right] .
$$

Where $F$ is the confluent hypergeometric function. To a good approximation we can write

$$
\varepsilon=0.79 \frac{\sigma^{3}}{L_{\mathrm{r}}} \cdot c_{\mathrm{c}}
$$

where $L_{\mathrm{r}}$ is the largest of the pulse length and the beam width, and $c_{\mathrm{c}}$ is a correction factor very close to 1 .

As noted, Eqs. (14) and (24) are conceptually very different. Why should this be?

The answer to this question can be seen in the diagrammatic sketch shown in Fig. 1. This diagram shows a schematic representation of the spectrum, as well as the weighting effect of the radar beam. It also emphasizes the fact that 


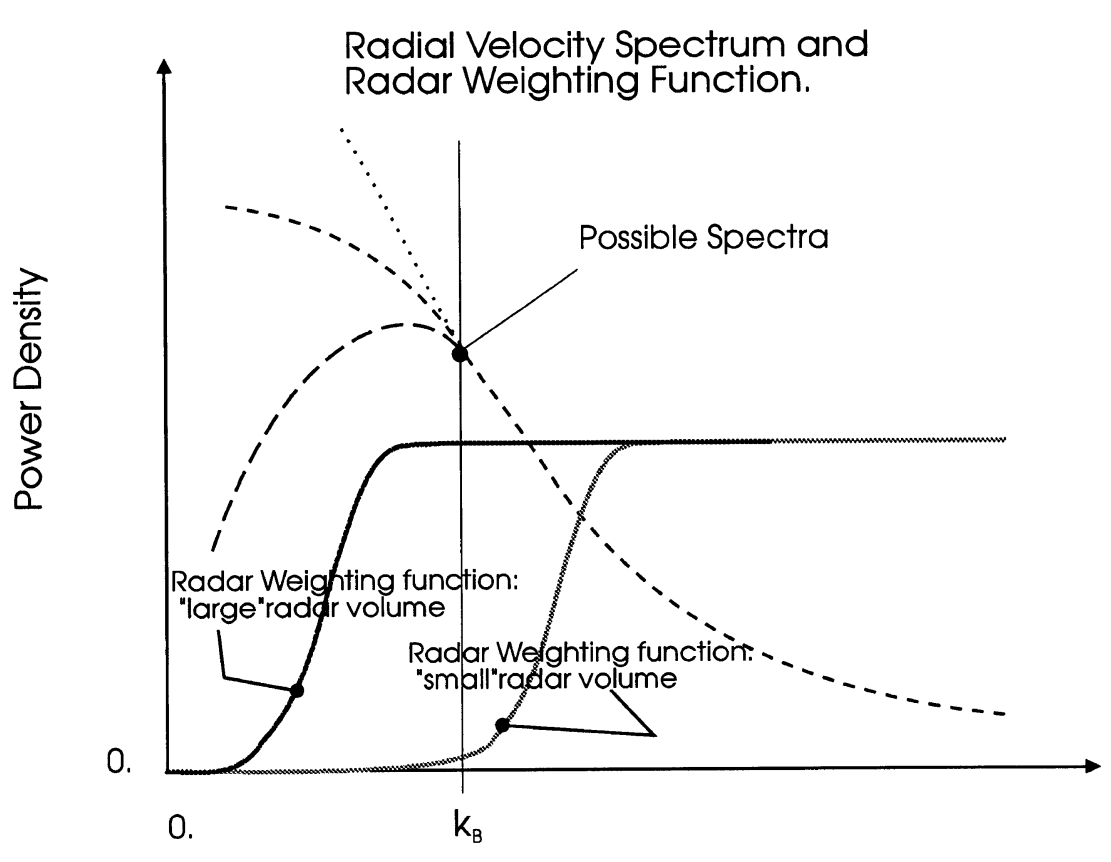

Wavenumber (k)

Fig. 1. Graphs showing the radial velocity spectra and radar weighting functions for different assumed spectra and radar volumes.

the spectrum may have a "roll-over" at small wavenumbers, where a "roll-over" refers to a moderately abrupt but smooth change in slope. This may be evident as a "knee" in the spectrum, or even a local peak. Whether such a "rollover" exists depends on what one assumes about the nature of the low wave number spectrum (often the gravity wave spectrum) at the scales close to the turbulence regime. It also depends on which radial velocities are being measured-a vertically pointed radar measures principally the vertical fluctuating motions, whilst a horizontally pointed radar measures largely horizontal components of motion. In our discussion we are primarily considering near-vertical beams, which are the main modes used for middle atmosphere studies.

This "roll-over" is what causes the Labitt formalism to break down. Labitt assumed that the Kolmogoroff spectral form (i.e. $\propto k^{-5 / 3}$ ) continued down to $k=0$, and this is why his integral involves $L_{\mathrm{r}}$. Such an assumption may be valid if the radar is used to point its beam horizontally (as is the case, for example, with the meteorological NEXRAD radars). However, if this "roll-over point" in the spectrum occurs at wave-numbers which are greater than the lowest wave-numbers corresponding to the radar volume, then the integral begins to involve $L_{\mathrm{B}}$. For most middle atmosphere radars, near-vertical beams are used, so this latter possibility is likely.

Figure 1 shows how this comes about. The integrand involves a product of the spectrum and the weighting function, and it is seen that if the weighting function is that for a "small" radar volume, and we follow it from large $k$ back to small $k$, then the weighting drops to zero before $k_{\mathrm{B}}$ is encountered. Thus the integral does not involve any portion of the spectrum at $k$ values below $k_{\mathrm{B}}$. However, in the case labelled "large radar volume", the radar weighting function does not start to approach zero (reading from the right) until the spectrum has entered the "buoyancy" regime. Thus the nature of the spectrum in this low wave-number end begins to affect the integral.

The situation is also indicated diagrammatically, but in a different way, in Fig. 2. In the first case, we show a region of turbulence with the radar volume being substantially smaller than the largest scales of turbulence. In this case, we expect the Labitt formula to apply. However, the other diagrams (b, c, and d) show cases where some part of the radar volume exceeds (or is at least comparable with) the largest scales of the turbulence. In this case, we expect the formula with an $L_{\mathrm{B}}$ dependence to apply.

Thus Labitt has ignored the small wavenumber departure from the inertial range law. However, we should also point out that Eq. (12) is also only a crude approximation, since it assumes that the spectrum drops abruptly to zero at the wavenumber $k_{\mathrm{B}}$. Therefore both approaches have their weaknesses-Eq. (12) is mathematically crude, while Eq. (17) is mathematically rigorous but ignores the true smallwavenumber spectral variation. It makes sense to combine the formalisms, to try and take advantage of both of their strengths.

In the following section, we will put the concepts discussed above into a mathematical setting, and demonstrate that our expectations are valid. In fact, we will show that the largest cross-volume length of the radar volume must be less than one half of the buoyancy scale for the Labitt formula to apply-in all other cases, the formula involving $L_{\mathrm{B}}$ is more appropriate. 


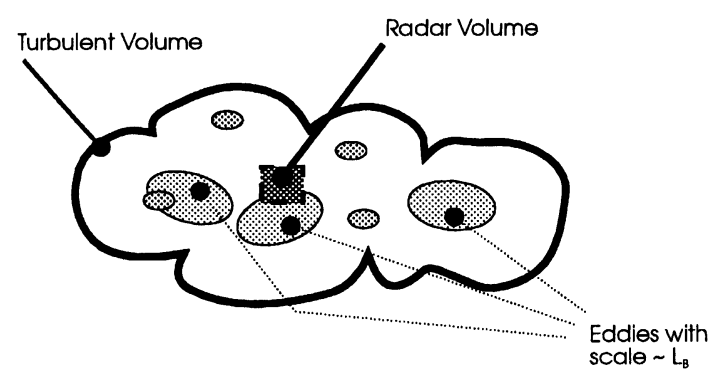

a.

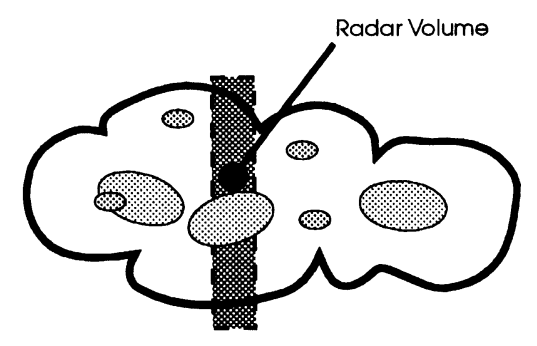

c.

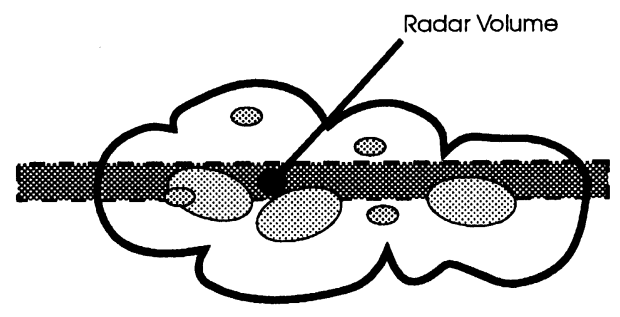

b.

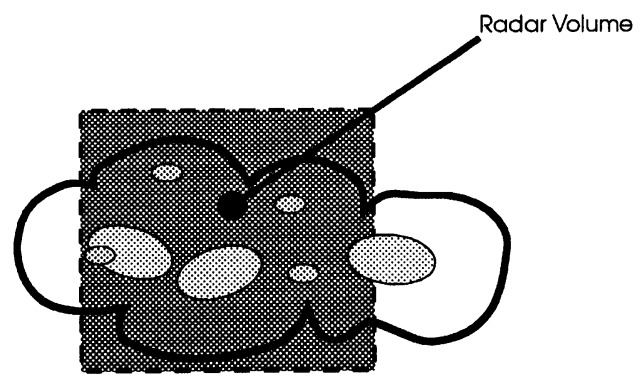

d.

Fig. 2. Different possible relations between the radar volume and a patch of turbulence. Only in the first case is the behaviour of the turbulence spectrum at small $k$ (i.e. below $k_{\mathrm{B}}$ ) unimportant in determing the relation between $\varepsilon$ and $\sigma$. In all other cases, the relation between $\varepsilon$ and $\sigma$ has a $k_{\mathrm{B}}$ dependence.

\section{Combining the Buoyancy Part of the Spectrum within the Labitt-Formalism}

We will now re-address Eqs. (17) and (18), but this time we will permit $E(k)$ to have a "roll-over" point at low wavenumber. We will see that this substantially changes Eq. (24), and in fact makes the result appear more like (14) in many cases.

To begin, we propose the following possible shape of the spectrum at small $k$, (as discussed by Hocking, 1996a):

$$
E(k)=\alpha \varepsilon^{2 / 3} \frac{k^{-5 / 3}}{\left[1+\chi_{k}\left(k / k_{\mathrm{B}}\right)^{n}\right]}
$$

where $k_{\mathrm{B}}=2 \pi / L_{\mathrm{B}}$ and where the value of $n$ determines the form of the low wave-number part of the spectrum. The value of $\chi_{k}$ affects the relative positions of the low-wavenumber "roll-over point" in the spectrum and the quantity $k_{\mathrm{B}}$. Hocking (1996a), used the special cases $n=-3$ and $-4 / 3$, because they represent extreme examples of the possible spectral forms, and thus set reasonable limits on our formulae. They correspond to cases with $E(k) \propto k^{+4 / 3}$ and $k^{-1 / 3}$ at small $k$ respectively. Examples are shown diagrammatically in Fig. 3 for the case of $\chi_{k}=1.0$. Clearly the "knee" (or "peak" for the case $n=-3$ ) is close to the value of $k_{\mathrm{B}}$, so henceforth we will use $\chi_{k}=1.0$ as a reasonable approximation, although we recognize that future more detailed experimental studies might give slightly different values for this parameter. At present, however, there are insufficient experimental data to better define $\chi_{k}$.

As noted prior to Eq. (18), this equation implicitly assumes an isotropic spectrum. However, this is not entirely unreasonable for the cases we wish to consider. In addition, for a vertically directed beam it is principally the vertical velocity

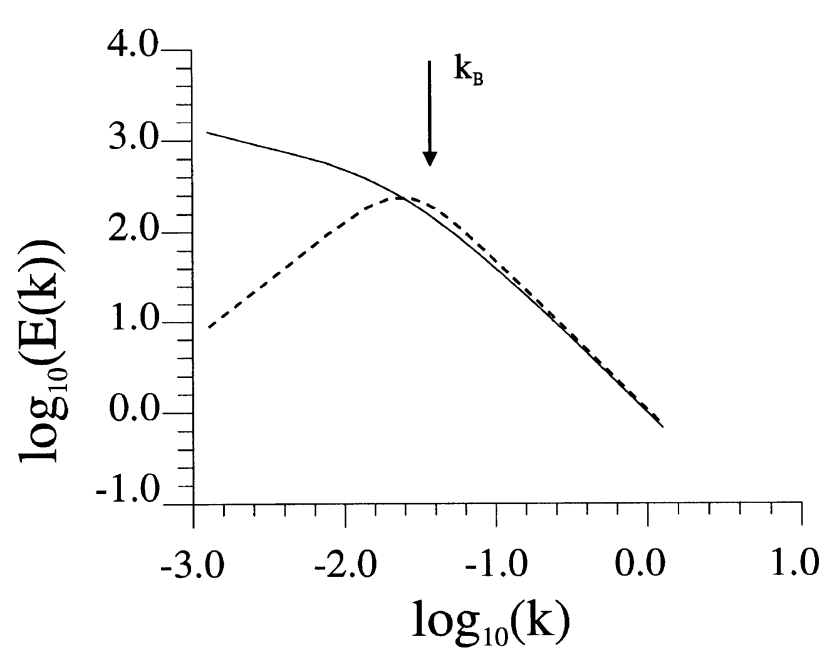

Fig. 3. Representative forms for the turbulence spectrum $E(k)$, including typical possible variations at small $k$. Specifically these graphs show Eq. (25), for $n=-3$ and $-4 / 3$. The $n=-3$ case corresponds to a power law of the type $k^{4 / 3}$ at small $k$, and is represented by the broken line; the $n=-4 / 3$ case corresponds to a power law of the type $k^{-1 / 3}$ at small $k$, and is represented by the solid line. In both cases the buoyancy scale is the same and equals $250 \mathrm{~m}$; the corresponding wavenumber lies very close to the peak in the broken curve (from Hocking, 1996a).

fluctuations which are important, so as long as $E(k)$ is chosen so as to produce a reasonable vertical velocity spectrum, any lack of isotropy is not too critical to our arguments. It should also be recognized that we only seek to place reasonable limits on the relation between spectral widths and the energy dissipation rates, so great accuracy in specifying $E(k)$ is not required-indeed, it is presently not available 


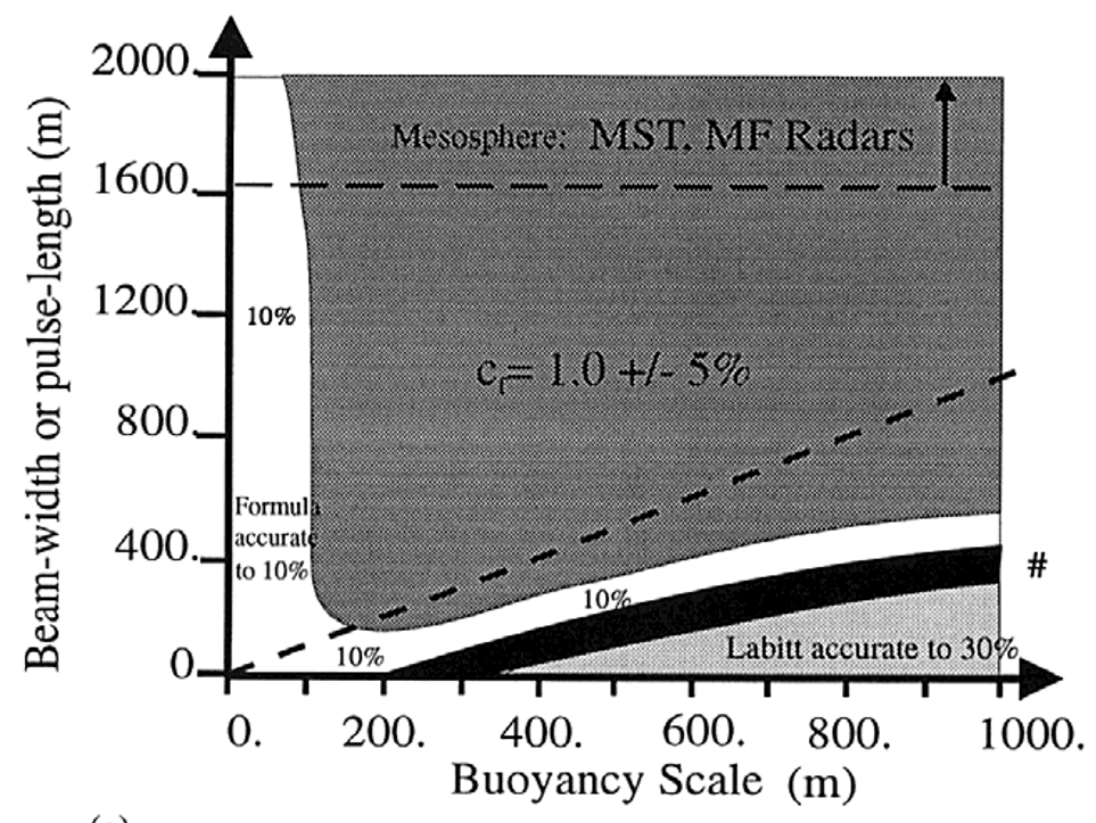

(a)

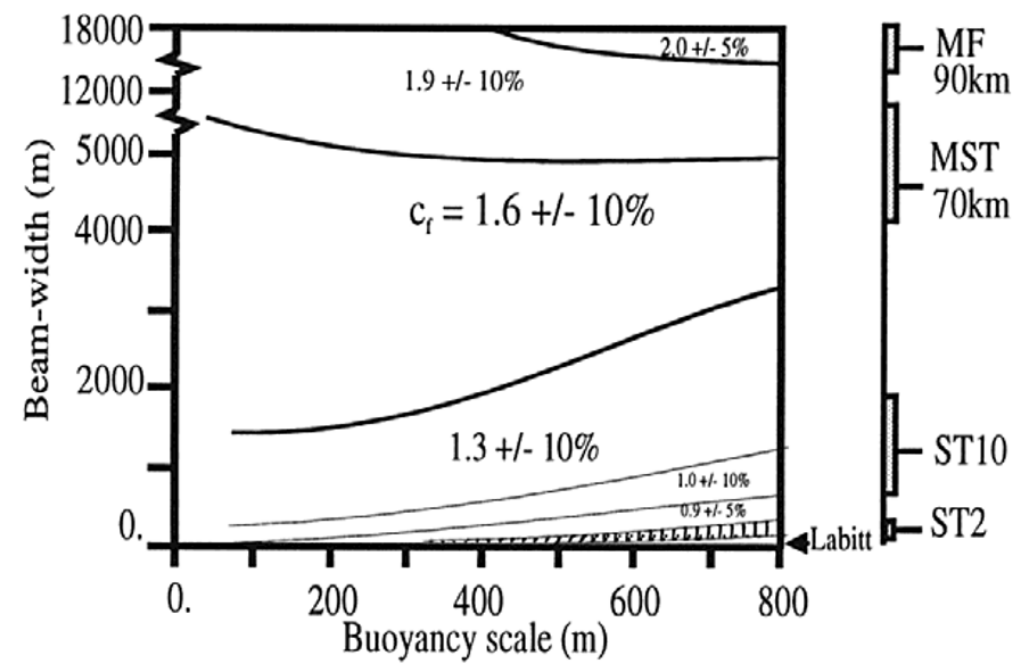

(b)

Fig. 4. These two graphs show corrections to the formula $\varepsilon \simeq\left(0.45 \sigma^{2} \omega_{\mathrm{B}}\right)$ in radar applications. Specifically, they show values for $c_{\mathrm{f}}$ in the expression $\varepsilon \simeq\left(0.45 \sigma^{2} \omega_{\mathrm{B}}\right) c_{\mathrm{f}}^{-2 / 3}$, for cases of (a) $n=-3$ and (b) $n=-4 / 3$. Cases where the "Labitt formalism" should be used are also indicated. In case (b), the scale on the right side indicates the approximate heights at which the appropriate beam-widths shown on the left apply, assuming an angular beam-width of typically 2 to 5 degrees.

as an experimentally measured quantity, which is another reason why we have taken this more approximate course of action.

We now must determine

$$
\begin{aligned}
\Upsilon= & \int_{\theta=0}^{\pi} \int_{k=0}^{\infty} \sin ^{3} \theta \frac{k^{-5 / 3}}{\left[1+\left(k / k_{\mathrm{B}}\right)^{n}\right]} \\
& \times\left[1-\mathrm{e}^{-k^{2}\left[a^{2} \sin ^{2} \theta+b^{2} \cos ^{2} \theta\right]}\right] d k d \theta .
\end{aligned}
$$

Hocking (1996a) has numerically integrated this expression for a wide range of combinations of $k_{\mathrm{B}}$ and pulse length. With respect to the case $n=-3\left(E \propto k^{4 / 3}\right.$ at small $\left.k\right)$, he found the following. Provided that the larger of the radar pulse-length and beam-width exceeds one half of the buoy- ancy scale, then to very good accuracy, $\Upsilon$ can be represented closely by the following expression:

$$
\Upsilon=\left(0.45 L_{\mathrm{B}}\right)^{2 / 3} .
$$

Hence, using Eq. (21) we obtain the relation

$$
\varepsilon=3.3 \frac{\sigma^{3}}{L_{\mathrm{B}}}=0.47 \sigma^{2} \omega_{\mathrm{B}}
$$

This compares very favourably to the estimates made in the earlier literature, in which the equation $\varepsilon=0.45 \sigma^{2} \omega_{\mathrm{B}}^{2}$ has been given e.g. see Eq. (16). Figure 4(a) shows a contour graph in which a measure of the ratio of the true value of $\varepsilon$ relative to the above formula is shown for various beam 
widths and various buoyancy scales $L_{\mathrm{B}}$. The area in which the Labitt formula is accurate is also highlighted; note that throughout most of the region described by this graph the dependence of $\varepsilon$ on $L_{\mathrm{B}}$ is very important and the Labitt formalism is generally not valid. As experimental support for this prediction, it is noteworthy that Bohne (1981: abstract), who attempted to use the Labitt formalism to produce radar measurements of $\varepsilon$, and then compared them with measurements made in-situ, found that he could only make useful estimates of $\varepsilon$ for those cases in which the radar pulse length was less than one half of the buoyancy scale. The reason for the inaccuracy of the radar measurement in these cases of small $L_{\mathrm{B}}$ was almost certainly because Eq. (28) should have been used, rather than the Labitt approach.

Let us now turn to the case of $n=-4 / 3$. In this case the spectrum goes as $k^{-1 / 3}$ as $k$ tends to 0 . Then in fact numerical integration of Eq. (26) over a wide range of possible buoyancy scales and possible pulse lengths and beam widths gives the following expression:

$$
\varepsilon=3.3 \frac{\sigma^{3}}{L_{\mathrm{B}}} \frac{1}{c_{\mathrm{f}}}=0.47 \sigma^{2} \omega_{\mathrm{B}}\left[\frac{1}{c_{\mathrm{f}}}\right]^{2 / 3}
$$

where $c_{\mathrm{f}}$ is a correction factor. Even in this case, where the buoyancy range runs somewhat smoothly into the inertial range, but where the energy involved in the buoyancy range is higher than that in the inertial range, it can be seen that the dependence on $L_{\mathrm{B}}$ is still significant and the expression given by Labitt is generally not appropriate.

Figure 4(b) shows the value of the correction factor over a wide range of beam widths and buoyancy scales. Note that the region in which the Labitt formalism is approximately correct is indicated and is clearly only a small portion of the region. For MST radars the Labitt equation is almost never valid and the previous expression (29) is correct. Furthermore, the correction factor is a fairly slowly varying term which varies from as small as 0.9 for very small beam widths and very long buoyancy scales up to a factor of as high as 2 for very broad beam widths (widths of several kilometres). The correction factor is dependent on the characteristics of the particular radar being used, but it is not a strong function of the radar parameters, and a reasonable estimate of it can be made in almost all circumstances.

Thus in summary, we see that the correct equations to use for converting $\sigma^{2}$ from radar measurements (after removal of beam and shear-broadening (e.g. Hocking 1983; Nastrom, 1997)) is in fact Eq. (29) with correction factors as shown in Figs. 4(a) or 4(b) (depending on the nature of the spectrum as it goes from the turbulent regime to the gravity wave regime).

We have thus unified the two sets of possible formulae discussed earlier, and also demonstrated when each applies. This is an important result for future applications of radar measurements in studies of turbulence strengths using radars.

We now move on to discussion of the other methods for measurement of atmospheric turbulence. The previous discussion concentrated on measurements of velocity fluctuations, whereas the next section will look in more detail at scalar parameters.

\section{Scalar Spectral Methods for Measuring $\varepsilon$}

In this section, we will consider measurements of scalar quantities like potential refractive index, neutral fluctuations, and ion and electron densities, and discuss how they may be used to infer $\varepsilon$. We will concentrate on two main areasfirstly, the ways in which radar can be used to measure refractive index fluctuations, and then the ways in which direct in-situ measurements of spectra can be employed to determine $\varepsilon$.

The first case relates to application of Eq. (10), and we now wish to address the questions we have raised in relation to that equation. To begin, we first recognize that $C_{n}^{2}$ is a measure of refractive index fluctuations, and refractive index fluctuations are related more to potential energy perturbations and less to kinetic energy fluctuations. Thus the relationship between $C_{n}^{2}$ and $\varepsilon$ depends on the ratios of potential to kinetic energy. Since this ratio is Richardson-number dependent, it might not be surprising to find that $\gamma$ could depend on the Richardson number. Nevertheless, there have been documents in which it has been assumed that $\gamma$ is indeed a constant, and for a while this was accepted as standard. In the next section, we will re-examine the rather complex history associated with $\gamma$. Again, we remind the reader that the terminology of "constant" for $C_{n}^{2}$ is very misleading, but is maintained here for historical reasons. In the following section, we consider $C_{n}^{2}$ not as a true constant, but simply as a variable which parameterizes the degree of potential refractive index fluctuation in a turbulent patch. Our main point of discussion will be the dynamical parameter $\gamma$. We emphasize that the following discussion relates both to radar measurements of turbulence strengths using absolute backscatter techniques, as well as in-situ measurements of ion, electron and neutral density fluctuations.

\subsection{The "constant" $\gamma$}

Despite the above expectation about a Richardson-number dependence of $\gamma$, for a while this dependence was all but ignored in the literature, and $\gamma$ was indeed taken as a constant. Examples include Van Zandt et al. (1978, 1981), Gage (1980), as well as Hocking (1985), Thrane et al. (1985, 1987), Lübken et al. (1987) and Blix et al. (1990). Note that in the last four cases, it was not actually $C_{n}^{2}$, the potential refractive index gradient structure "constant", which was measured, but rather one of the neutral, ion or electron density structure "constants". Nevertheless, the same principle applied, and in each case the $R_{i}$ dependence of $\gamma$ was not properly considered.

This is not to say that the non-constancy of $\gamma$ was unknown, but rather it was fully appreciated only in fields other than middle atmospheric ones. Examples of references which demonstrate a Richardson number dependence include Ottersten (1969), Crane (1980), and Gossard et al. (1982, 1985, 1987). However, for middle atmosphere applications many of these early references were not utilized. To be fair, however, the $R_{i}$ dependency was often not recognized because it was impossible to employ it, simply because measurements of $R_{i}$ with sufficient resolution were not possible. More recent papers like Hocking (1992), Blix (1993) and Hocking and $\mathrm{Mu}$ (1997) have given due recognition to the more realistic $R_{i}$ dependence in middle atmosphere applications, but are again constrained by the inability of cur- 
rent techniques to make measurements of $R_{i}$ with sufficient resolution to be useful. Nevertheless, the recognition of this dependence is important from a conceptual viewpoint, which is why we pursue it here.

We will now recap some of the earlier papers which noted that $\gamma$ was not in fact a constant. Ottersten (1969) gave

$$
\gamma=\frac{1}{a^{2}}\left(\frac{1-R_{f}}{R_{i}}\right)=\frac{1}{a^{2} P_{r}}\left(\frac{P_{r}-R_{i}}{R_{i}}\right)
$$

where $a^{2}$ is a constant, $R_{i}$ is the gradient Richardson number, $R_{f}$ is the flux Richardson number, and $R_{f}=P_{r}^{-1} R_{i}, P_{r}$ being the turbulent Prandtl number. $P_{r}$ is defined as $K_{\mathrm{m}} / K_{\mathrm{T}}$, where $K_{\mathrm{m}}$ and $K_{\mathrm{T}}$ are the turbulent momentum and heat diffusion coefficients respectively.

Gossard et al. $(1982,1984)$ present an expression in which $\gamma$ effectively obeys

$$
\gamma=\frac{1}{B_{\theta}} \frac{P_{r}-R_{i}}{R_{i}}
$$

where $B_{\theta}=3.2$.

Hocking (1992) assumed to first order a turbulent Prandtl number of unity and obtained, via energy balance arguments, the following expression for $\gamma$;

$$
\gamma=\frac{3}{22} \frac{\left|1-R_{i}\right|}{\left|R_{i}\right|}
$$

The ratios of potential to kinetic energy storage as a function of $R_{i}$, as deduced by Hocking (1992), are shown graphically in Fig. 5.

We therefore recognize that even when the $R_{i}$ dependence of $\gamma$ is understood, there is not general agreement about the details of the relationship. Different authors have produced different relationships, and we cannot resolve these differences here. Our preference is to use Eq. (32).

If Richardson number measurements are not available, then a value of

$$
\gamma=0.4
$$

is recommended as a reasonable compromise, since it corresponds approximately with a Richardson number of 0.25 according to (32). We therefore see that we are once again

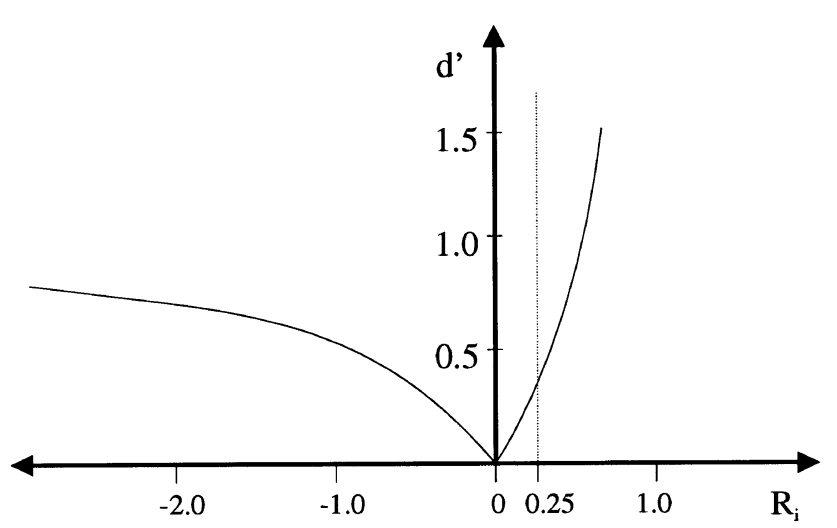

Fig. 5. The ratio of the potential energy and kinetic energy spectral densities, $d^{\prime}$, plotted as a function of the Richardson number, $R_{i}$. Note that the ratio tends to infinity as $R_{i}$ approaches 1 , and tends to 1 as $R_{i}$ approaches negative infinity (from Hocking, 1992). returning to an assumption of a constant value for $\gamma$, but this approach is adopted simply because it is often not possible to measure $R_{i}$ with sufficient resolution. It is fairest to think of this as a mean value for $\gamma$. It is often the best we can do, but is definitely an inferior approach to proper use of $R_{i}$ in determining $\gamma$.

\subsection{An alternative way to determine $\varepsilon$ using spectral fitting around the spectral knee}

Because of uncertainties in regard to application of the pre-

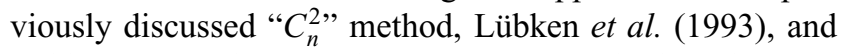
Lübken (1997) developed an alternative method for determination of $\varepsilon$. This method still employs direct measurements of scalar spectra, but in a different manner to that described in the previous section. It has been well-known for many years that if one can measure $\eta$, the Kolmogoroff microscale, then one can determine $\varepsilon$ through the relation (7). The kinematic viscosity $v$ is usually taken from empirical atmospheric models. The major difficulty is determination of $\eta$ accurately, because $\varepsilon$ is proportional to $\eta$ to the fourth power. For example, an error in $\eta$ of a factor of 2 means an error in $\varepsilon$ of a factor of 16. Traditionally $\eta$ has been determined by finding the inner scale, $\ell_{0}$, and then determining $\eta$ through (8) using an assumed value for $c_{4}$. (e.g. Watkins et al., 1988). The value of $c_{4}$ depends on whether one is measuring velocity fluctuations, ion fluctuations, neutral fluctuations or whatever, as seen earlier.

This method fell from favour, however, because there was too much uncertainty in determining $\ell_{0}$. Different extrapolation schemes produced different values. Lübken has recently attempted to solve this difficulty by fitting a carefully prescribed function to the Fourier-spectrum of the time series of neutral density fluctuations measured by a moving rocket (expressed as a function of the spectral angular frequency $\omega$ ) viz.

$$
\begin{aligned}
W(\omega)= & \frac{\Gamma(5 / 3) \sin (\pi / 3)}{2 \pi v_{\mathrm{r}}} \cdot C_{n}^{2} \cdot f_{\alpha} . \\
& \times \frac{\left(\omega / v_{\mathrm{r}}\right)^{-5 / 3}}{\left[1+\left\{\left(\omega / v_{\mathrm{r}}\right) / k_{0}^{\prime}\right\}^{8 / 3}\right]^{2}} .
\end{aligned}
$$

An angular frequency of $\omega$ corresponds to a spatial scale in the turbulence along the track of the rocket with "wavelength" equal to $2 \pi v_{\mathrm{r}} / \omega$. Here, $\Gamma(5 / 3)=0.90167 ; v_{\mathrm{r}}$ is the rocket speed; $f_{\alpha}=2.0$, and $k_{0}^{\prime}=2 \pi / \ell_{0}^{\prime}$, where $\ell_{0}^{\prime}$ is a length scale closely related to $\ell_{0}$. The denominator in the last multiplicative term was introduced as an attempt to allow the inertial range to run smoothly into the viscous range, and is somewhat ad-hoc. Because this is so, it is necessary to exercise some care in the meaning of $\ell_{0}^{\prime}$. Lübken et al. (1993), and Lübken (1997) made the (unproven) assumption that $\ell_{0}^{\prime}=\ell_{0}$. We wish to emphasize that because this is not yet proven, it represents a possible source of systematic error in the following discussions, and we will distinguish between $\ell_{0}^{\prime}$ and $\ell_{0}$ in our discussions here-in, although we recognize that Lübken et al. did not. An alternative way to write (34) would be

$$
\begin{aligned}
W(\omega)= & \frac{\Gamma(5 / 3) \sin (\pi / 3)}{2 \pi v_{\mathrm{r}}} \cdot C_{n}^{2} \cdot f_{\alpha} . \\
& \times \frac{\left(\omega / v_{\mathrm{r}}\right)^{-5 / 3}}{\left[1+\chi_{L}\left\{\left(\omega / v_{\mathrm{r}}\right) / k_{0}\right\}^{8 / 3}\right]^{2}} .
\end{aligned}
$$




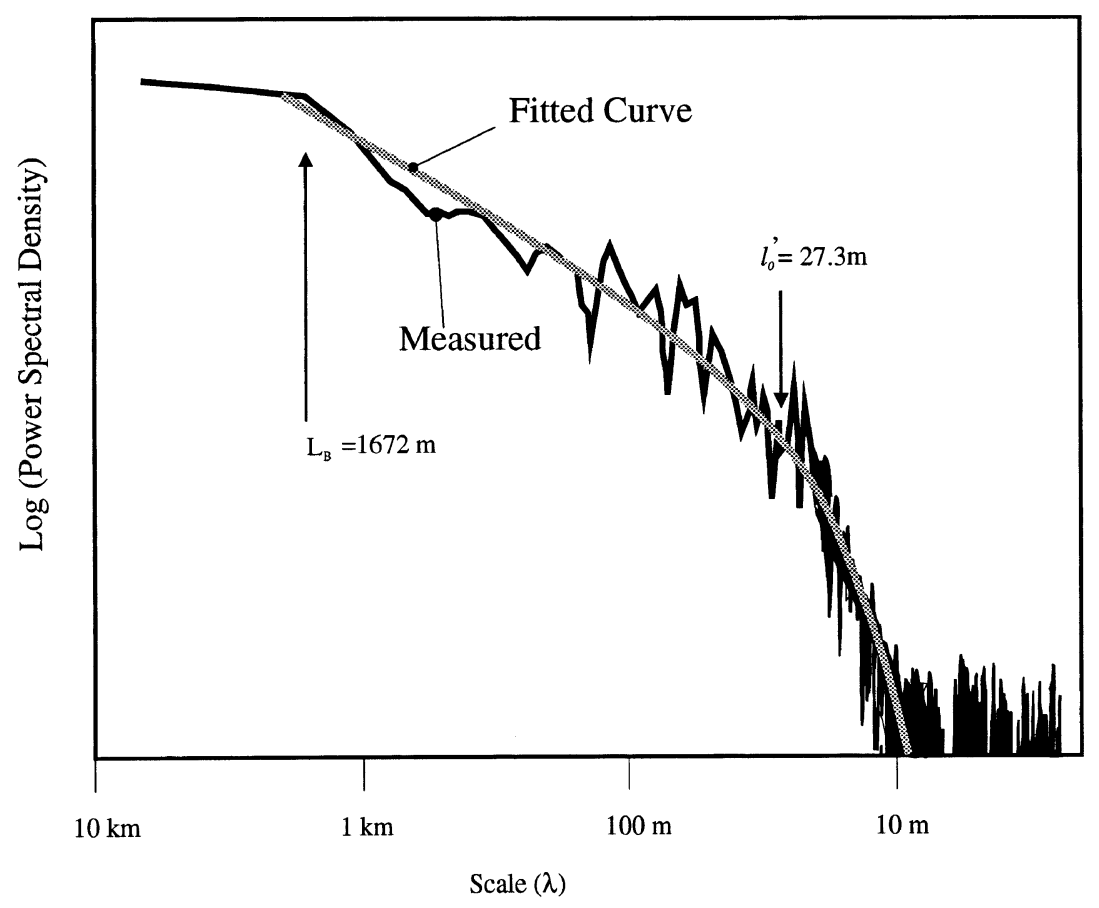

Fig. 6. Experimental and fitted spectra for rocket measurements of neutral density fluctuations. The smooth curve shows a fit assuming a Heisenberg model in the viscous range. Buoyancy and inner scales are also shown (adapted from Lübken, 1997).

where $k_{0}=2 \pi / \ell_{0}$, and the term $\chi_{L}$ accounts for the fact that the spectral "knee" need not occur directly at a wavenumber of $k_{0}$.

By fitting this functional form to the measured spectra, Lübken et al. (1993), and Lübken (1997) were able to determine $\ell_{0}^{\prime}$ to fairly high accuracy. The value of $\ell_{0}^{\prime}$ can be determined independently of $C_{n}^{2}$ and $f_{\alpha}$. They then assumed that $\ell_{0}^{\prime}$ is proportional to $\ell_{0}$, and so used a variation on (8) viz.

$$
\ell_{0}^{\prime}=c_{4}^{\prime} \eta
$$

They used $c_{4}^{\prime}=9.90$ to get $\eta$, and thence determined $\varepsilon$. This choice required some knowledge about the Prandtl number, and there is some uncertainty in this regard. Lübken et al. (1993) and Lübken (1997) used 0.82, whilst Hill and Clifford (1978) suggest 0.72 . The latter result is the correct choice if it is recognized that the temperature spectra and the neutral density spectra are identical in form. An example of the measured and fitted spectra is shown in Fig. 6.

However, it is appropriate at this juncture that we make some comments about the function $W(\omega)$. This function is designed to describe both the inertial range of the spectrum as well as the viscous range, plus the transition between them. It is proportional to $\omega^{-7}$ at large $\omega$, which limits its usefulness to some degree. For example, if one requires the variance of the third derivative of the spatial fluctuations, (as is sometimes sought in turbulence studies), then it involves an integral over all $\omega$ of $W$ multiplied by $\omega^{6}$, which is an integral of $\omega^{-1}$, and is therefore infinite. Higher order derivatives have similar infinities. Indeed, Heisenberg's original proposal for a $\omega^{-7}$ form at high wavenumbers was criticized by, for example, Batchelor, for reasons like this. Furthermore, Heisenberg's formula was really only supposed to apply to energy spectra, whereas Lübken et al. have adapted it to scalar spectra. The possibility of such infinite integrals places some limits on the usefulness of this particular function; if this functional form is indeed used, it is necessary that the user places some sort of artifical limit on the integrals, or assumes that the spectrum changes form yet again at some point well into the viscous range.

Indeed, the optimal choice of $W(\omega)$ requires additional discussion, and should at this stage be considered indeterminate. Lübken found by experimentally fitting the data to different functions that the so-called "Heisenberg" theoretical form described by Eqs. (34) and (35) gave the best fit, although his original papers also discussed a model due to Tatarskii (1971) for the viscous range. However, we have noted doubts about the suitability of the Heisenberg form. Another possibility which well deserves examination is the temperature spectrum of Hill and Clifford (1978). It should be recognized that within turbulence in the free air, the fluctuations in temperature and the fluctuations in density should have the same form, since neutral fluctuations due to pressure perturbations are negligible, so this is an excellent candidate. Nevertheless, for now we recognize that Lübken's preference is to use Eq. (34). We recognize that the chief new contribution from Lübken et al. (1993) and Lübken (1997) to measurements of turbulence was to develop a formalism whereby $\ell_{0}^{\prime}$ could be determined using all of the available spectrum, thereby (hopefully) producing higher accuracy.

This method was then used extensively by Lübken (1997) to determine a climatology of $\varepsilon$. An example will be discussed shortly in regard to Fig. 8. The method appears to be moderately reliable, although it should be emphasized that the assumption that $\ell_{0}^{\prime}=\ell_{0}$ is still unproven; this can lead to systematic errors in $\varepsilon$. Questions about the correct choice of 


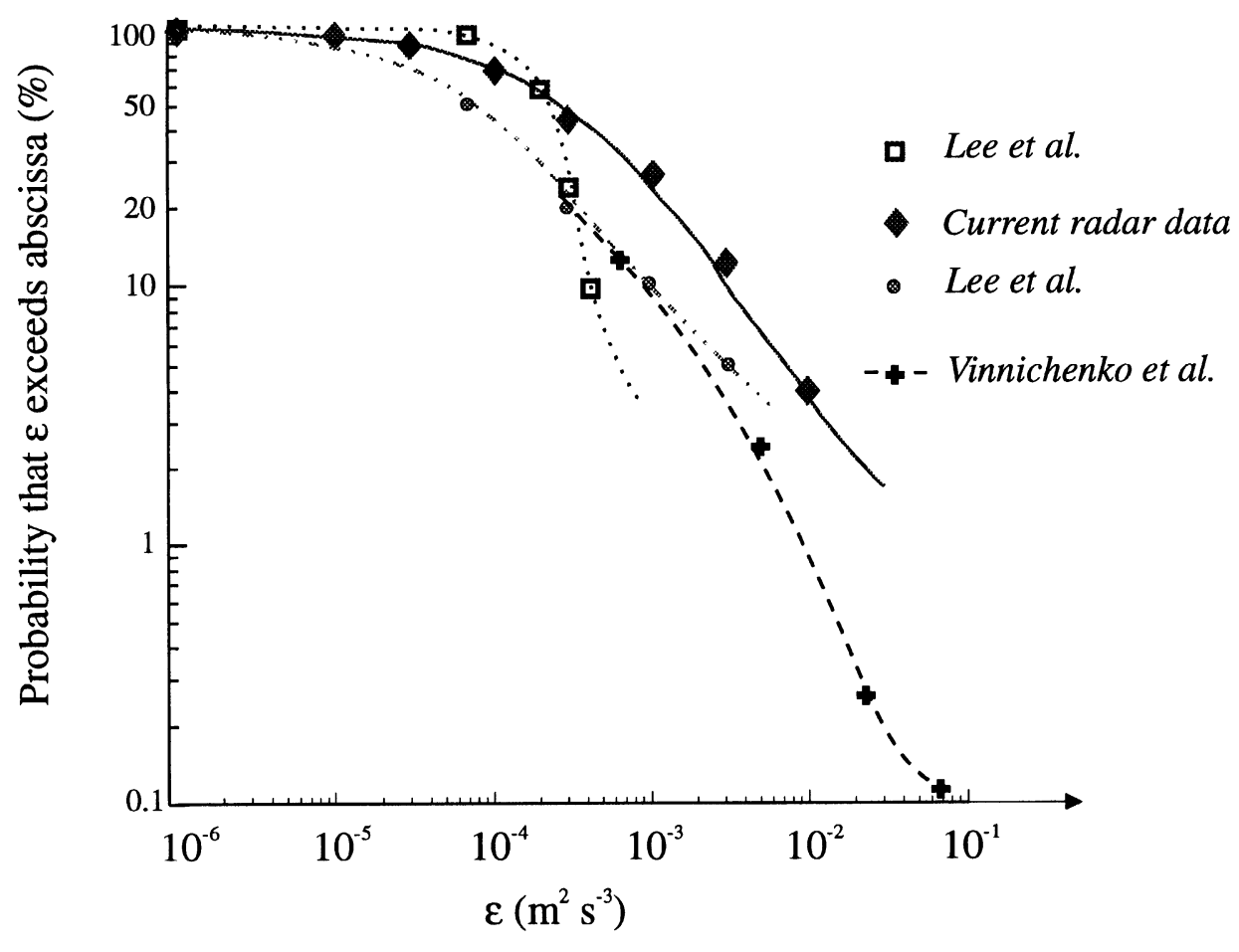

Fig. 7. Cumulative graph of $\varepsilon$ in the troposphere (from Hocking and Mu, 1997), using radar data and the theory embodied in Eqs. (10) and (33), as well as various in-situ measurements. Data are compared to Lee et al. (1988) and Vinnichenko et al. (1973).

the Prandtl number have also been noted above. Additionally, because $\varepsilon$ varies as the fourth power of $\ell_{0}^{\prime}$, even small errors in estimating $\ell_{0}^{\prime}$ can lead to considerable errors in $\varepsilon$. However, even despite these problems, the method remains one of the more commonly used for rocket studies of turbulence. It is only possible to guess at the effects of these systematic errors, although we would hope that the method gives accuracies which are correct to within a factor of 2 .

\subsection{Application of the new $C_{n}^{2}$ formula to some in-situ data}

In this section, we wish to intercompare the two approaches described in Subsections 6.1 and 6.2, since they have been two of the main approaches to determinations of $\varepsilon$ by rocket techniques. Previous comparisons have not always shown good agreement, but in each case we have noted recent developments and adjustments, so it will be of interest to see how the two different techniques now compare after these new developments are considered.

The formulae presented in Subsection 6.1, which involve the more proper use of $\gamma$, have been tested in at least a couple of cases, and seem to produce somewhat better estimates than do those which do not properly consider the Richardsonnumber dependence of this quantity. We shall illustrate some of these, but it should nevertheless be borne in mind that even the tests shown here are not really definitive, and more tests are unquestionably needed. In particular, in these tests we have had to assume that $\gamma=0.4$, whereas it would be much nicer to use actual measured values of the Richardson number made at scales of a few tens to hundreds of metres.

The first such test is shown in Fig. 7, which summarizes results from Hocking and $\mathrm{Mu}$ (1997), using tropospheric data. This shows a cumulative distribution of energy dissipa- tion rates measured by various techniques, including radar. Whilst the data were taken at different sites, and on different occasions, the overall agreement is quite reasonable. Values obtained by radar and shown here, for example, show broadly better agreement that do those which do not use this more recent theory.

A more interesting comparison comes about by examining the same data using two different analysis techniques. We have chosen the rocket data obtained by Thrane et al. (1985, 1987), Lübken et al. (1987), and Blix et al. (1990), which have been nicely tabulated in those references. We have converted the energy dissipation rates produced by these authors back to effective structure constants (analagous to $C_{n}^{2}$ but in this case they were ion or electron density or neutral density structure constants), and then re-determined $\varepsilon$ using Eqs. (10) and (33). We used $F$ equal to 1 , because when using in-situ data there is no need to concern ourselves about an incompletely filled measuring volume - the data are recorded at very high resolution by the moving probe, and the measuring instruments have volumes much smaller than the size of any turbulent patch. We have then compared the new data to estimates of $\varepsilon$ obtained by Lübken (1997) using his "spectralknee"formalism (see the previous section). The results are shown in Fig. 8; we have concentrated on the region above $80 \mathrm{~km}$ altitude. The most important line is that for winter, since most of the raw data used were taken in Autumn and Winter (specifically October 1987, November 1980, January 1984 and February 1984; see references cited above). The solid circles (theory presented here-in) seem to show better agreement with Lübken (1997) than do the filled squares. Therefore it seems that data produced with the newer version of (10), using (33), provide broadly better consistency with 


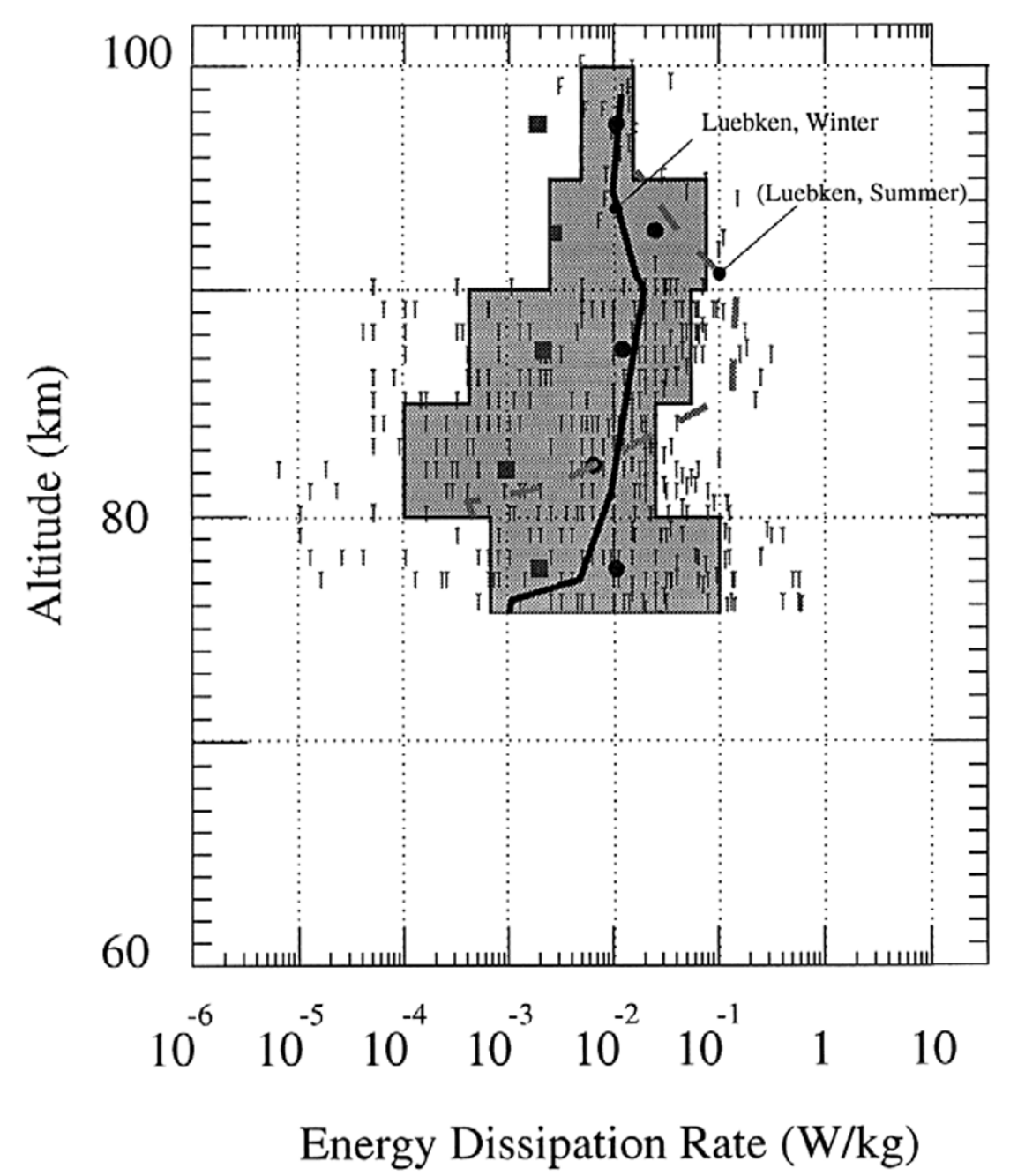

Fig. 8. Energy dissipation rates from Thrane et al. (1985), Lübken et al. (1987) and Blix et al. (1990), produced after rescaling according to Eqs. (10) and (33). Rescaled raw data are shown by the symbols "T". The filled squares show median values of $\varepsilon$ due to the original authors, whilst the solid circles show median values using the newer theory. The left and right borders of the filled area shows $16 \%$ and $84 \%$ percentiles using the newer theory. The solid lines show estimates for summer and winter due to Lübken (1997), using his procedure for fitting spectra to the data.

the methods described in Subsection 6.2 than do the earlier methods.

\section{The Relation between Diffusion and Energy Dissipation Rates}

The issue of the relation between the rates of diffusion and the rate of energy dissipation in the atmosphere is another area which is often oversimplified. It is often assumed that (11) applies, and that measurements of $\varepsilon$ immediately enable determination of the rate of vertical diffusion, $K$. Authors vary in their assumed values of $c_{2}$, but most (with the possible exception of McIntryre, 1989) generally agree that the value lies between 0.2 and 1.25 (e.g. Fukao et al., 1994; Lilly et al., 1974; Weinstock, 1981). We will not dwell too much on the actual value of $c_{2}$ here; it is premature to specify it more precisely than has been done here, although a value of 0.8 is commonly used.

A more important matter here is not what $c_{2}$ is, but rather whether (11) applies at all. The methods by which diffusion can take place are far more complex than simple threedimensional turbulent diffusion. The reasons for this lie in two main facts; first, turbulence is very intermittent both temporally and spatially, and very often occurs in thin layers in the middle atmosphere. These thin layers are often separated by regions which are either only weakly turbulent or even laminar. Secondly, the processes which induce diffusion can themselves be scale dependent.

These factors mean that there are several ways in which diffusion can occur. Table 1 summarizes some of these processes, and we will now elaborate briefly upon them.

The first important factor is the spatial and temporal intermittency. This effect has been demonstrated in Hocking (1991, 1996b), after adaptation from Desaubies and Smith (1982). These authors show how an ensemble of gravity waves can act together to produce regions of instability separated in height by regions of stability, with layer thicknesses of a few tens of metres out to a kilometre or so. Examples of experimental studies of such layering are also discussed there-in.

The consequences of this intermittency are important. They mean, for example, that we must revisualize how largescale turbulent diffusion takes place. An important proposal 
Table 1. This table shows some of the various processes which are normally grouped together as "diffusive" processes in the atmosphere. Classical turbulent diffusion is only one such process, and at large scales is not necessarily even one of the most important. At intermediate scales (500 $\mathrm{m}$ to $3 \mathrm{~km}$ ), all of these processes occur, but we have left question marks here to indicate that it is uncertain just which of all these processes dominates in this regime.

\begin{tabular}{|c|c|c|}
\hline Scale & Momentum & Constituents/temperature \\
\hline$<500 \mathrm{~m}$ & $K \propto \frac{\varepsilon}{\omega_{\mathrm{B}}^{2}}$ & $K \propto \frac{\varepsilon}{\omega_{\mathrm{B}}^{2}}$ \\
\hline \multirow[t]{3}{*}{$500 \mathrm{~m}$ to $3 \mathrm{~km}$} & all processes & all processes \\
\hline & described above and below- & described above and below- \\
\hline & but which dominates? & but which dominates? \\
\hline \multirow[t]{6}{*}{$>3 \mathrm{~km}$} & "Classical turbulent diffusion" & "Classical turbulent diffusion" \\
\hline & Stochastic Layering & Stochastic Layering \\
\hline & Quasi-horizontal diffusion & Quasi-horizontal diffusion \\
\hline & (Slant-wise convection) & (Slant-wise convection) \\
\hline & & Stokes Diffusion \\
\hline & + other? & + other? \\
\hline
\end{tabular}

due to Dewan (1981) and Woodman and Rastogi (1984) suggested that the random occurrence of layers produces a Monte Carlo type of intermittent diffusion. In this model, diffusion is not a continuous process, but a step-wise one. First one layer of turbulence forms around a particle of interest, purely due to chance. Turbulent transport of this particle then takes place, possibly to the edge of the layer, or until the layer dies out. At this time the particle remains fairly stationary, since molecular diffusion is assumed to be very small. Then at a later time, another turbulent layer forms around the particle, and further transport over the depth of that layer is now possible. This process repeats itself over and over. Thus the factors which control the large-scale diffusion are not simply the rates of diffusion across individual layers, but the frequency of occurrence and depth of individual layers (this process is illustrated diagramatically in Fig. 2 of Hocking, 1991). Any determinations of effective diffusion coefficients must take this into account. Proper modelling of the effects of this intermittency remains an important area of research.

Other consequences of the intermittency of turbulence include the possibility that the average rates of diffusivity of momentum and heat may be different, and that the Prandtl number may exceed 1, and perhaps be in the range of 1 to 3 (Fritts and Dunkerton, 1985). This is to say that if one parameterizes the rate of heat transport as $K_{\mathrm{T}}(\partial \bar{\theta} / \partial z)$, where $\partial \bar{\theta} / \partial z$ is the mean potential temperature gradient, ignoring the effects of the wave, then the effective coefficient which must be used to describe the rate of diffusion is less than it would be if we properly included the effect of the wave in $\partial \bar{\theta} / \partial z$. This is not so for momentum diffusion, because ' $u$ ' and ' $w$ ' are not in phase quadrature. Fritts and Dunkerton (1985) have proposed this process as a way to explain the conclusions of Strobel et al. (1987), in which these authors claim that the turbulent Prandtl number is somewhat in excess of unity in the atmosphere.

Another important means of vertical diffusion is quasihorizontal diffusion along tilted isopleths. It is well known that horizontal diffusion at large scales is a much faster pro- cess than vertical diffusion. If the mean gradients are tilted, then this horizontal diffusion attains a vertical component, and can lead to an effective vertical mixing. Admittedly a particle which starts at an altitude of $z \mathrm{~km}$, and finally achieves a height of $z+\zeta \mathrm{km}$, may also have drifted horizontally a distance equal to perhaps hundreds of times $\zeta$, but nevertheless this still produces an effective vertical mixing.

Another important process which can produce significant diffusion is so-called "Stokes Diffusion", as proposed by Walterscheid and Hocking (1991) and Hocking and Walterscheid (1993). These authors have shown that even a linear combination of Boussinesq waves produces a diffusive-like effect on particles over periods of many hours, and whilst this process is not as strong as classical turbulence in causing diffusion at scales of a few tens to hundreds of metres, it becomes a major diffusive effect when applied at scales of many hours. This is because it is not affected by the intermittency of turbulence, and acts just as strongly in laminar regions as it does in turbulent ones. This process is especially important for diffusion of constituents. If the waves are damped, the diffusive effect becomes even stronger, especially if the damping induces particles to cross between contours of constant potential tempearture; in this case, Stokes diffusion may also be important for momentum diffusion. As noted, Table 1 summarizes some of these processes.

Therefore we conclude this section by simply noting that the relation between rates of diffusion and energy dissipation is not simple, and in fact is both scale and species dependent. This is still an area which deserves much research, and the key point to note is that previous visualizations and parameterizations of these processes have been grossly oversimplified in the past. Diffusion is scale dependendent, and the types of diffusion coefficients which a global-scale modeller might use may be very different (usually larger) than the ones which might be needed to describe small scale mixing in the atmosphere. 


\section{Conclusion}

Some of the constants traditionally used in turbulence theory, and indeed some classical interpretations, have been re-examined. The basis for these formulae have been discussed, showing how some of these constants arise. Appropriate formulae for application of radar and in-situ measurements of turbulence have been presented, including recommendations for the most appropriate constants where possible. Where necessary, oversimplifications in current thinking about turbulence have also been pointed out. Without question, though, all current measurements of energy dissipation rates in the middle atmosphere have uncertainties of some type; a major goal in the next few years should be to develop instrumentation which can directly measure velocity fluctuations in-situ down to scales within the viscous range. Only then will it be possible to unambiguously interpret the spectra, and determine turbulent energy dissipation rates with precision.

Acknowledgments. The careful comments and suggestions of two anonymous reviewers were of great help in the final preparation of this document. This work was supported in part by the Natural Sciences and Engineering Research Council of Canada.

\section{Appendix A. Velocity Structure Functions}

The following appendices summarizes the main structure functions and spectra used in turbulence theory, without proof or derivation.

The first type of function which we will discuss that is commonly used to describe turbulent phenomena is the socalled Structure Function. There are several of these, but the main ones are $D_{\|}$and $D_{\perp}$, which are defined in the following way;

$$
D_{\|}(r)=\overline{\left|u_{\|}(\underline{x}+\underline{r})-u_{\|}(\underline{x})\right|^{2}}
$$

and

$$
D_{\perp}(r)=\overline{\left|u_{\perp}(\underline{x}+\underline{r})-u_{\perp}(\underline{x})\right|^{2}},
$$

where we imagine traversing the turbulent medium in a straight line and taking point measurements along the way. "Parallel" components refer to measurements of the velocity components with directions parallel to the direction of traverse, and "perpendicular" components refer to velocity components perpendicular to this direction. Isotropy has been assumed in this definition, which is why we consider $D$ to depend only on the magnitude $r$ of the vector $\underline{r}$.

Occasionally a 3-D form of the structure function is sometimes used, viz.

$$
D_{\text {tot }}(\underline{r})=\overline{|\underline{u}(\underline{x}+\underline{r})-\underline{u}(\underline{x})|^{2}},
$$

where the vector difference between displaced components is used. Because there are two perpendicular components, and one parallel component, we may write

$$
D_{\text {tot }}=D_{\|}+2 D_{\perp} \text {. }
$$

For inertial range, homogeneous, Kolmogoroff-style turbulence, we have the following relations.

$$
D_{\|}=C_{v}^{2} r^{2 / 3}
$$

where $C_{v}^{2}=C \varepsilon^{2 / 3}$, and $C$ is close to 2.0 (e.g. Caughey et al., 1978; Kaimal, 1976). In addition,

$$
\begin{aligned}
D_{\perp} & =\frac{4}{3} C_{v}^{2} r^{2 / 3}, \\
D_{\text {tot }} & =\frac{11}{3} C_{v}^{2} r^{2 / 3} .
\end{aligned}
$$

There are also a variety of spectral forms which are used as tools in turbulence studies.

\section{Appendix B. Spectral Forms for Velocity Measure- ments}

A variety of spectra are used for turbulence studies. These all have different purposes, and are summarized below for Kolmogoroff-type inertial-range turbulence.

The first important expression is

$$
\mathcal{F}(\underline{k})=A \varepsilon^{2 / 3} k^{-11 / 3}
$$

where $k=|\underline{k}|$ is the length of the vector $k$, (and so takes values between 0 and infinity), and $A=\frac{11 \Gamma\left(\frac{8}{3}\right) \sin \left(\frac{\pi}{3}\right)}{24 \pi^{2}} C \simeq$ $0.061 C$, (Tatarskii, 1971). This is a full three-dimensional function describing the total kinetic energy per unit cell size (due to all three velocity components) in a cell of size $d^{3} k$ at the end of a vector $\underline{k}$ originating from the origin. For homogeneous isotropic turbulence this function is isotropic. Pictorially one can visualize this as a solid sphere in $\left(k_{x}, k_{y}, k_{z}\right)$ space which has highest density at the centre, and decreasing density as $|k|$ increases, where the density represents $\mathcal{F}$.

Because this function is isotropic, it is often integrated over a shell of radius $k$ to give a new expression which is

$$
E(k)=4 \pi k^{2} \mathcal{F}=\alpha \varepsilon^{2 / 3} k^{-5 / 3}
$$

where $\alpha=4 \pi A=\frac{11 \Gamma\left(\frac{8}{3}\right) \sin \left(\frac{\pi}{3}\right)}{6 \pi} C=0.76655 C$ (e.g. see Tatarskii, 1971; Batchelor, 1953). Note that we will largely follow Batchelor's symbol-usage in this document: For example, we use $E(k) d k$ to represent the total energy in a shell in $k$-space of thickness $d k$, as does Batchelor, whereas Tatarskii $(1961,1971)$ uses the symbol $E$ to represent the function which we have called $\mathcal{F}$.

If we use $C=2.0$, then we have

$$
E(k)=1.53 \varepsilon^{2 / 3} k^{-5 / 3} .
$$

Different authors use different values for the constant 1.53-anything between 1.35 and 1.53 are common. Note, however, that if one adjusts this constant then the constant $C$ also needs adjustment. I prefer to use $C=2.0$ because it has at least been measured with good accuracy in the lower atmosphere (e.g. Caughey et al., 1978)

These equations are fairly simple to understand. However, there are more complex variants. An important adjunct (and in fact a more fundamental expression) is the equation

$$
\Phi_{i j}(\underline{k})=\frac{E(k)}{4 \pi k^{4}} \cdot\left(k^{2} \delta_{i j}-k_{i} k_{j}\right)
$$

which describes the three-dimensional cross-spectrum between the velocity components in the " $i$ " direction and the " $j$ " direction, where " $i$ or $j=1$ " mean the $x$ direction, " $i$ 
or $j=2$ " mean the $y$ direction and " $i$ or $j=3$ " mean the $z$ direction. The values $k_{1}, k_{2}$ and $k_{3}$ may take both positive and negative values. Note that $k$ is the length of the vector from the origin to the point $\left(k_{1}, k_{2}, k_{3}\right)$ in $k$-space, and so $k^{2}=k_{1}^{2}+k_{2}^{2}+k_{3}^{2}$.

For each of these spectra there is a related covariance function; for example,

$$
\Phi_{i \ell}(\underline{k})=\frac{1}{8 \pi^{3}} \int_{-\infty}^{\infty} \int_{-\infty}^{\infty} \int_{-\infty}^{\infty} e^{-\jmath \underline{k} \cdot \underline{\xi}} R_{i \ell}(\underline{\xi}) \underline{d \xi}
$$

where $R_{i \ell}$ is the aoutovariance function corresponding to $\Phi_{i \ell}$ and where $J=\sqrt{-1}$ in this expression. We will not discuss these various covariance functions in much detail here; the reader is referred to to Tatarskii $(1961,1971)$, Batchelor (1953) or Lumley and Panofsky (1964) for more elaborate discussions.

For cases of isotropic turbulence, we can integrate $\Phi_{i j}$ around a shell of radius $k$ to give (e.g. Batchelor, 1953, p. 35)

$$
\Psi_{i j}(k)=\oiint \Phi_{i j}(\underline{k}) k^{2} d \Omega_{k}
$$

For homogeneous, isotropic turbulence, we therefore have

$$
\Psi_{i j}(k)=4 \pi k^{2} \Phi_{i j}(\underline{k}) .
$$

$E(k)$ relates to the $\Psi_{i j}$ (and hence to the $\Phi_{i j}$ ) via the relation

$$
E(k)=\frac{1}{2}\left(\Psi_{11}(k)+\Psi_{22}(k)+\Psi_{33}(k)\right) .
$$

Notice the factor $\frac{1}{2}$; this is introduced so that the integral over all $k$ (i.e. from $k=0$ to $k=\infty$ ) gives the kinetic energy per unit mass, $\frac{1}{2} \overline{v_{\text {tot }}^{2}}$. $E(k)$ is unique in this regardother spectra have normalizations which do not involve this factor of $\frac{1}{2}$. For example,

$$
\int_{0}^{\infty} \Psi_{11}(k) d k=\overline{u_{1}^{2}}
$$

where $u_{1}$ refers to the velocity component in the $x$ direction.

Sometimes (B.8) is also written as

$$
E(k)=\frac{1}{2} \oiint_{k_{i} k_{i}=k^{2}} \Phi_{i i}(\underline{k}) k^{2} d \Omega_{k},
$$

where the subscript ' $i i$ ' means sum the three terms $\Phi_{11}, \Phi_{22}$ and $\Phi_{33}$ (e.g. Lumley and Panofsky, 1964, p. 28).

The above spectra are useful from a conceptual viewpoint, but are often hard to determine experimentally, since they require a full three-dimensional description of the turbulent field in all three velocity components. That is, they require knowledge of all three velocity components at all points in space. This is often difficult (if not impossible) to measure.

Therefore, we also look for spectral analogues to the structure functions which were described earlier for a onedimensional pass through the turbulent field.

To begin, if we have a detector which moves in a straight line through a patch of turbulence, and it records the velocity components parallel to the direction of motion (in analogy to the process described in connection with Eqs. (A.1) to (A.3)), and then we Fourier transform the resultant spatial series, we obtain (for Kolmogoroff turbulence) the function

$$
\Theta_{11}\left(k_{1}, 0,0\right)=\alpha_{11}^{\prime} \varepsilon^{2 / 3}\left|k_{1}\right|^{-5 / 3}
$$

where $\alpha_{11}^{\prime}=\frac{9}{55} \alpha=0.1244 C$. This is in fact a onedimensional function which we will denote as $\phi_{p}$, viz.

$$
\phi_{p}\left(k_{1}\right)=\alpha_{11}^{\prime} \varepsilon^{2 / 3}\left|k_{1}\right|^{-5 / 3} .
$$

It is important to note that this is not the same as $\Phi_{11}\left(k_{1}, 0,0\right)$. Whilst both refer to spectral densities along the $x$ axis, $\Phi_{11}\left(k_{1}, 0,0\right)$ refers to spectral densities due only to "waves" with the phase-fronts aligned perpendicular to the $x$ axis. On the other hand, $\Theta_{11}\left(k_{1}, 0,0\right)$ (and $\left.\phi_{p}\left(k_{1}\right)\right)$ refer to the spectral density at wavenumber $k_{1}$ due to contributions of "waves" of all orientations which cross the $x$ axis. These concepts are fundamentally different. In fact,

$$
\Theta_{i j}\left(k_{1}, 0,0\right)=\iint \Phi_{i j}\left(k_{1}, k_{2}, k_{3}\right) d k_{2} d k_{3} .
$$

Likewise, if we find the spectrum for the velocity components perpendicular to the direction of motion during this traverse, we produce

$$
\phi_{t}\left(k_{1}\right)=\Theta_{22}\left(k_{1}, 0,0\right)=\alpha_{22}^{\prime} \varepsilon^{2 / 3}\left|k_{1}\right|^{-5 / 3}
$$

where $\alpha_{22}^{\prime}=\frac{4}{3} \alpha_{11}^{\prime}$.

Additionally, for the choice of $C=2.0$ described above, we have

$$
\begin{gathered}
\phi_{p}\left(k_{1}\right)=\Theta_{11}\left(k_{1}, 0,0\right)=0.25 \varepsilon^{2 / 3}\left|k_{1}\right|^{-5 / 3} \\
-\infty<k_{1}<\infty \\
\phi_{t}\left(k_{1}\right)=\Theta_{22}\left(k_{1}, 0,0\right)=0.33 \varepsilon^{2 / 3}\left|k_{1}\right|^{-5 / 3} \\
-\infty<k_{1}<\infty
\end{gathered}
$$

In the case of isotropic turbulence, there is no preferred axis, so that these formulae are not restricted to any particular axis.

Because of the obvious symmetry, many experimentalists often "fold" their spectral densities at negative wavenumbers over onto their positive ones, and so do not differentiate between positive and negative signs for the wavenumber. Then we obtain the following functions:

$$
\begin{array}{r}
\phi_{p}^{\prime}\left(k_{\alpha}\right)=0.50 \varepsilon^{2 / 3} k_{\alpha}^{-5 / 3} \\
0<k_{\alpha}<\infty, \\
\phi_{t}^{\prime}\left(k_{\alpha}\right)=0.67 \varepsilon^{2 / 3} k_{\alpha}^{-5 / 3} \\
-\infty<k_{\alpha}<\infty
\end{array}
$$

where $k_{\alpha}$ are absolute values of wavenumbers along the direction of travel of the probe.

Note that Eqs. (B.11), (B.12), (B.14), and (B.15) to (B.18), have " $k^{-5 / 3}$ " laws, but so does (B.2). However, these equations are conceptually different; (B.2) represents an integration over a shell of radius $k$ in three-dimensional $k$-space, whilst (B.15) to (B.18) represent spectra determined by a probe moving in a straight line through the turbulence. Nevertheless, it is a common mistake for novice researchers to confuse the two spectra, when they speak of the " $k$ " which can lead to the propagation of considerable confusion. It is important to conceptually distinguish these spectra. 


\section{Appendix C. Scalar Structure Functions and Spec- tra}

In some studies of turbulence, it is not information about the velocity fluctuations which are sought, but rather density fluctuations associated with certain tracers. One must be careful to choose a "good" tracer-certainly quantities which react chemically with their surrounds will not obey the following equations (e.g. see Hocking, 1985).

The structure function is described as

$$
D_{\zeta}(r)=\overline{|\zeta(\underline{x}+\underline{r})-\zeta(\underline{x})|^{2}}
$$

where $\zeta$ represents the scalar concentration. Kolmogoroff inertial range turbulence this is given by

$$
D_{\zeta}(r)=C_{\zeta}^{2} r^{2 / 3} .
$$

The first important spectral form is $\Phi_{\zeta}(\underline{k})$, which is the full three-dimensional spectral density function. For Kolmogoroff turbulence, it is given by

$$
\Phi_{\zeta}(\underline{k})=0.033 C_{\zeta}|\underline{k}|^{-11 / 3}
$$

in the inertial range. The nearest analogy to this spectrum for the velocity case is the function $\mathcal{F}$ from Eq. (B.1); $\Phi_{\zeta}$ should not be confused with $\Phi_{i j}$ from Eq. (B.4), although the notations look similar. This convention may seem just a little confusing, but is maintained here as a result of historical precedent.

This function has been chosen to be normalized so that

$$
\int_{-\infty}^{\infty} \int_{-\infty}^{\infty} \int_{-\infty}^{\infty} \Phi_{\zeta}(\underline{k}) d \underline{k}=\overline{\left(\zeta^{\prime}\right)^{2}}
$$

Then for locally isotropic, homogeneous we define

$$
E_{\zeta}(k)=4 \pi k^{2} \Phi_{\zeta}(k)
$$

or

$$
E_{\zeta}(k)=0.132 \pi C_{\zeta}^{2}|\underline{k}|^{-5 / 3}=0.415 C_{\zeta}^{2}|\underline{k}|^{-5 / 3} .
$$

The function $E_{\zeta}$ is very analagous to the function $E$ in Eq. (B.2).

Finally, we present the spectrum seen if we record along a straight line. This is the spectrum which a probe moving through a patch of turbulence would measure, and is very similar to $\phi_{p}$ from Eqs. (B.12) and (B.14) in the section on velocity spectra. This is given by

$$
S_{\zeta}\left(k_{1}\right)=\int_{-\infty}^{\infty} \int_{-\infty}^{\infty} \Phi_{\zeta}(\underline{k}) d k_{2} d k_{3}
$$

which, for the case of Kolmogoroff turbulence, becomes

$$
\begin{aligned}
S_{\zeta}(k)= & 0.125 C_{\zeta}^{2}|\underline{k}|^{-5 / 3} \\
& -\infty<k<\infty .
\end{aligned}
$$

The function $S_{\zeta}$ has strong similarities with $\phi_{p}$ in Eq. (B.15). If we fold negative wavenumbers onto positive, we obtain

$$
\begin{array}{r}
S_{\zeta}^{\prime}(k)=0.25 C_{\zeta}^{2} k^{-5 / 3} \\
0<k<\infty .
\end{array}
$$

Again (as for the velocity spectra), note that (C.6) and (C.9) both involve a " $k^{-5 / 3}$ " law, but the spectra are conceptually different.

\section{Appendix D. $C_{n}^{2}$ and $\varepsilon$}

The energy dissipation rate is related to the potential refractive index structure constant by

$$
\bar{\varepsilon}=\left(\gamma \overline{C_{n}{ }^{2}} \frac{\omega_{\mathrm{B}}^{2}}{F^{1 / 3}} M^{-2}\right)^{3 / 2}
$$

where $\omega_{\mathrm{B}}$ is the Väisälä-Brunt frequency. The parameter $F$ represents the fraction of the radar volume which is filled by turbulence, while $\gamma$ is discussed in more detail in the main body of the text.

The "potential refractive index gradient" is given in the troposphere and stratosphere by

$$
\begin{aligned}
M= & -77.6 \times 10^{-6} \frac{P}{T}\left(\frac{\partial \ln \theta}{\partial z}\right) \\
& \times\left[1+\frac{15500 q}{T}\left(1-\frac{1}{2} \frac{\partial \ln q / \partial z}{\partial \ln \theta / \partial z}\right)\right]
\end{aligned}
$$

where $z$ is height, $\theta$ is the potential temperature, $q$ is the specific humidity, $T$ is the absolute temperature and $P$ is the atmospheric pressure in millibars. The term in square brackets was denoted as $\chi$ by Van Zandt et al., 1978; indeed this particular form of the equation was first introduced by these authors. Note that $\chi$ tends to 1 as the humidity terms tend to zero.

In the ionosphere, where humidity is no longer important but electron density plays a crucial role, we have

$$
M=\frac{\partial n}{\partial N}\left[\frac{N}{\theta}-\frac{d N}{d z}+\frac{N}{\rho} \cdot \frac{d \rho}{d z}\right]
$$

where again we have used the symbol $\theta$ for potential temperature and $N$ is the electron density. The term $\rho$ is the neutral density. The function $\frac{\partial n}{\partial N}$ needs to be determined from electro-ionic theory (e.g. Sen and Wyller, 1960; Budden, 1965; Hocking and Vincent, 1982).

\section{References}

Barat, J., Some characteristics of clear air turbulence in the middle stratosphere, J. Atmos. Sci., 39, 2553-2564, 1982.

Batchelor, G. K., The Theory of Homogeneous Turbulence, 197 pp., Cambridge University Press, New York, 1953.

Batchelor, G. K., An Introduction to Fluid Dynamics, 615 pp., Cambridge University Press, Cambridge, New York, 1967.

Blix, T. A., In-situ studies of turbulence and mixing; problems and questions, in Coupling Processes in the Lower and Middle Atmosphere, Vol. 387 edited by E. V. Thrane, T. A. Blix and D. C. Fritts, NATO ASI series C, pp. 329-344, Kluwer Academic Publishers, 1993.

Blix, T. A., E. V. Thrane, and O. Andreassen, In situ measurements of the fine-scale structure and turbulence in the mesosphere and lower thermosphere by means of electrostatic positive ion probes, J. Geophys. Res., 95, 5533-5548, 1990.

Bohne, A. R., Radar Detection of Turbulence in Thunderstorms, 62 pp., Report \# AFGL-TR-81-0102 (ADA 108679), Air Force Geophys. Lab., Hanscom Air Force Base, MS, U.S.A., 1981.

Bohne, A. R., Radar detection of turbulence in precipitation environments, J. Atmos. Sci., 39, 1819-1837, 1982.

Budden, K. G., Effect of electron collisions on the formulas of magnetoionic theory, Radio Sci., 69D, 191-211, 1965.

Caughey, S. J., B. A. Crease, D. N. Asimakapoulos, and R. S. Cole, Quantitative bistatic acoustic sounding of the atmospheric boundary layer, $Q$. J. R. Meteorol. Soc., 104, 147-161, 1978.

Crane, R. K., A review of radar observations of turbulence in the lower stratosphere, Radio Sci., 15, 177-193, 1980.

Desaubies, Y. and W. K. Smith, Statistics of Richardson number and instability in oceanic internal waves, J. Phys. Oceanography, 12, 1245-1259, 1982. 
Dewan, E. M., Turbulent vertical transport due to thin intermittent mixing layers in the stratosphere and other stable fluids, Science, 211, 10411042, 1981.

Doviak, R. J. and D. S. Zrnic, Doppler Radar and Weather Observations, 458 pp., Academic Press, Orlanda, FL, 1984.

Fritts, D. C. and T. J. Dunkerton, Fluxes of heat and constituents due to convectively unstable gravity waves, J. Atmos. Sci., 42, 549-556, 1985.

Fukao, S., M. D. Yamanaka, N. Ao, W. K. Hocking, T. Sato, M. Yamamoto, T. Nakamura, T. Tsuda, and S. Kato, Seasonal variability of vertical eddy diffusivity in the middle atmosphere, 1 . Three-year observations by the middle and upper atmosphere radar, J. Geophys. Res., 99, 18973-18987, 1994

Gage, K. S., J. L. Green, and T. E. VanZandt, Use of Doppler radar for the measurement of atmospheric turbulence parameters from the intensity of clear air echoes, Radio Sci., 15, 407-416, 1980.

Gossard, E. E. and A. S. Frisch, Relationship of the variances of temperature and velocity to atmospheric static stability-application to radar and acoustic sounding, J. Clim. Appl. Meteorol., 26, 1021-1036, 1987.

Gossard, E. E., R. B. Chadwick, W. D. Neff, and K. P. Moran, The use of ground-based Doppler radars to measure gradients, fluxes and structure parameters in elevated layers, J. Appl. Meteorol., 21, 211-226, 1982.

Gossard, E. E., R. B. Chadwick, T. R. Detman, and J. Gaynor, Capability of surface-based clear-air Doppler radar for monitoring meteorological structure of elevated layers, J. Clim. Appl. Meteorol., 23, 474-485, 1984.

Gossard, E. E., J. Gaynor, R. J. Zamora, and W. D. Neff, Finestructure of elevated stable layers observed by sounder and in-situ tower sensors, $J$. Atmos. Sci., 42, 2156-2169, 1985.

Hill, R. J. and S. F. Clifford, Modified spectrum of atmospheric temperature fluctuations and its application to optical propagation, J. Opt. Soc. Am., 68, 892-899, 1978.

Hocking, W. K., On the extraction of atmospheric turbulence parameters from radar backscatter Doppler spectra-I: theory, J. Atmos. Terr. Phys., 45, 89-102, 1983.

Hocking, W. K., Measurement of turbulent energy dissipation rates in the middle atmosphere by radar techniques: a review, Radio Sci., 20, 1403$1422,1985$.

Hocking, W. K., Observation and measurement of turbulence in the middle atmosphere with a VHF radar, J. Atmos. Terr. Phys., 48, 655-670, 1986.

Hocking, W. K., The effects of middle atmosphere turbulence on coupling between atmospheric regions, J. Geomag. Geoelectr., 43, 621-636, 1991.

Hocking, W. K., On the relationship between the strength of atmospheric radar backscatter and the intensity of atmospheric turbulence, $A d v$. Space Res., 12(10), 207-213, 1992.

Hocking, W. K., An assessment of the capabilities and limitations of radars in measurements of upper atmosphere turbulence, Adv. Space Res., 17(11), 37-47, 1996a.

Hocking, W. K., Small scale dynamics of the upper atmosphere: experimental studies of gravity waves and turbulence, chapter 1.2.2 (invited) in The Upper Atmosphere, edited by W. Dieminger, G. K. Hartmann, and R. Leitinger, pp. 51-96, Springer-Verlag, Berlin, Heidelberg, New York, 1996b.

Hocking, W. K. and A. M. Hamza, A Quantitative measure of the degree of anisotropy of turbulence in terms of atmospheric parameters, with particular relevance to radar studies, J. Atmos. Sol.-Terr. Phys., 59, 10111020, 1997.

Hocking, W. K. and K. L. Mu, Upper and Middle Tropospheric Kinetic Energy Dissipation Rates from Measurements of $C_{n}^{2}$-Review of Theories, in-situ Investigations, and Experimental Studies using the Buckland Park Atmospheric Radar in Australia, J. Atmos. Terr. Phys., 59, 1779-1803, 1997.

Hocking, W. K. and R. A. Vincent, Comparative observations of D-region HF partial reflections at 2 and $6 \mathrm{MHz}, J$. Geophys. Res., 87, 7615-7624, 1982.

Hocking, W. K. and R. L. Walterscheid, The role of stokes' diffusion in middle atmospheric transport, NATO (North Atlantic Treaty Organization) publication in Coupling Processes in the Lower and Middle Atmosphere, (Series C: Mathematical and Physical Sciences, Vol. 387), edited by E. V. Thrane, T. A. Blix, and D. C. Fritts, pp. 305-328, Kluwer Academic Publishers, Dordrecht, Boston and London, 1993.

Kaimal, J. C., J. C. Wyngaard, D. A. Haugen, O. R. Cote, Y. Izumi, S. J. Caughey, and C. J. Readings, Turbulence structure in the convective boundary layer, J. Atmos. Sci., 33, 2152-2168, 1976.

Labitt, M., Some basic relations concerning the radar measurement of air turbulence, Mass. Inst. of Technol., Lincoln Lab., Work. Pap. 46WP5001, 1979.

Lee, Y., A. R. Paradis, and D. Klingle-Watson, Preliminary Results of the 1983 coordinated aircraft-Doppler weather radar turbulence experiment, volume I, Report \# DOT/FAA/PM-86/11 (A197894), 76 pp., Lincoln Lab., MIT, Lexington, Mass., U.S.A., 1988.

Lilly, D. K., D. E. Waco, and S. I. Adelfang, Stratospheric mixing estimated from high-altitude turbulence measurements, J. Appl. Meteorol., 13, 488 493, 1974.

Lübken, F.-J., Seasonal variation of turbulent energy dissipation rates at high latitudes as determined by in situ measurments of neutral density fluctuations, J. Geophys. Res., 102, 13441-13456, 1997.

Lübken, F.-J., U. Von Zahn, E. V. Thrane, T. Blix, G. A. Kokin, and S V. Pachomov, In-situ measurements of turbulent energy dissipation rates and eddy diffusion coefficients during MAP/WINE, J. Atmos. Terr. Phys., 49, 763-776, 1987.

Lübken, F.-J., W. Hillert, G. Lehmacher, and U. von Zahn, Experiments revealing small impact of turbulence on the energy budget of the mesosphere and lower thermosphere, J. Geophys. Res., 98, 20369-20384, 1993.

Lumley, J. L. and H. A. Panofsky, The Structure of Atmospheric Turbulence, 239 pp., John Wiley and Sons, New York, London, Sydney, 1964.

McIntyre, M. E., On dynamics and transport near the polar mesopause in summer, J. Geophys. Res., 94, 14617-14628, 1989.

Nastrom, G. D., Doppler radar spectral width broadening due to beamwidth and wind shear, Ann. Geophys., 15, 786-796, 1997.

Ottersten, H., Atmospheric structure and radar backscattering in clear air, Radio Sci., 4, 1179-1193, 1969.

Sen, H. K. and A. A. Wyller, On the generalization of the Appleton-Hartree magnetionic formulas, J. Geophys. Res., 65, 3931-3950, 1960.

Strobel, D. F., M. E. Summers, R. M. Bevilacqua, M. T. DeLand, and M. Allen, Vertical constituent transport in the mesosphere, J. Geophys. Res. 92, 6691-6698, 1987.

Tatarskii, V. I., Wave Propagation in a Turbulent Medium, 285 pp., McGrawHill, New York, 1961

Tatarskii, V. I., The Effects of the Turbulent Atmosphere on Wave Propagation, 472 pp., Keter Press, Jerusalem, 1971.

Thrane, E. V., Ø. Andreassen, T. Blix, B. Grandal, A. Brekke, C. R. Philbrick, F. J. Schmidlin, H. U. Widdel, U. Von Zahn, and F.-J. Lübken, Neutral air turbulence in the upper atmosphere observed during the Energy Budget Campaign, J. Atmos. Terr. Phys., 47, 243-264, 1985.

Thrane, E. V., T. A. Blix, C. Hall, T. L. Hansen, U. von Zahn, W. Meyer, P. Czechowsky, G. Schmidt, H.-U. Widdel, and A. Neumann, Small scale structure and turbulence in the mesosphere and lower thermosphere at high latitudes in winter, J. Atmos. Terr. Phys., 49, 751-762, 1987.

Van Zandt, T. E., J. L. Green, K. S. Gage, and W. L. Clark, Vertical profiles of refractivity turbulence structure constant: Comparison of observations by the Sunset radar with a new theoretical model, Radio Sci., 13, 819-829, 1978.

Van Zandt, T. E., K. S. Gage, and J. M. Warnock, An improved model for the calculation of profiles of and in the free atmosphere from background profiles of wind, Temperature and humidity, paper presented at 20th Conference on Radar Meteorology, Am. Met. Soc., Boston, Mass., Nov. 30-Dec. 3, 1981.

Vinnichenko, N. K., N. Z. Pinus, S. M. Shmater, and G. N. Shur, Turbulence in the Free Atmosphere (translated from Russian, translations editor J. A. Dutton), 263 pp., Consultants Bureau, NY, London, 1973.

Walterscheid, R. L. and W. K. Hocking, Stokes diffusion by atmospheric internal gravity waves, J. Atmos. Sci., 48, 2213-2230, 1991.

Watkins, B. J., C. R. Philbrick, and B. B. Balsley, Turbulence energy dissipation rates and inner scale sizes from rocket and radar data, J. Geophys. Res., 93, 7009-7014, 1988.

Weinstock, J., On the theory of turbulence in the buoyancy subrange of stably stratified flows, J. Atmos. Sci., 35, 634-649, 1978a.

Weinstock, J., Vertical turbulent diffusion in a stably stratified fluid, J. Atmos. Sci., 35, 1022-1027, 1978b.

Weinstock, J., Using radar to estimate dissipation rates in thin layers of turbulence, Radio Sci., 16, 1401-1406, 1981.

Woodman, R. F. and P. K. Rastogi, Evaluation of effective eddy diffusive coefficients using radar observations of turbulence in the stratosphere, Geophys. Res. Letts., 11, 243-246, 1984.

W. K. Hocking (e-mail: whocking@danlon.physics.uwo.ca) 